\title{
Secrecy Performance of Threshold-Based Cognitive Relay Network With Diversity Combining
}

\author{
Khyati Chopra, Ranjan Bose, and Anupam Joshi
}

\begin{abstract}
In the spectrum sharing mode, the transmitting power of the secondary user ( $\mathrm{SU}$ ) is optimally controlled, such that no additional interference occurs at the primary user (PU). In this paper, the secrecy outage performance is analyzed for such cognitive underlay decode-and-forward (DF) threshold-based relay network. The relayed and the direct signals are combined at the secondary eavesdropper and destination. Unlike other works to date, we have used both selection combining (SC) and maximal ratio combining (MRC) diversity schemes at the destination and eavesdropper with all possible permutations, and the best possible combination for cognitive system secrecy has been validated. Further, we consider threshold-based relaying where, the relay can successfully decode and re-transmit, only if the received signal-to-noise ratio (SNR) meets the particular threshold. We show that the diversity scheme, the threshold SNR, the secrecy rate, and the interference power all have a significant impact on cognitive system secrecy. We have also shown that the secrecy performance is more sensitive to changes in the quality of direct link between source to eavesdropper than source to destination, especially at the higher secrecy rates. The accuracy of the analytical expressions is corroborated by the computer simulation results.
\end{abstract}

Index Terms: Cognitive relay network, maximal ratio combining, selection combining, secrecy outage probability, threshold-based.

\section{Introduction}

$\mathrm{T}$ HE spectrum resources are exhausted due to the unparalleled increase in various wireless communication services. To opportunistically exploit the existing wireless spectrum, cognitive radio (CR) has emerged as a new communication paradigm for future high-coverage, high-capacity and multitier hierarchical networks $[1,2]$. The spectrum is shared without any performance degradation of primary users (PUs), by considering that the received interference remains below the predefined interference threshold level at the PU receiver (PU-Rx) [3-5]. The unused spectrum can be efficiently utilized by cognitive radio specifically in underlay $[5,6]$, for concurrently transmitting data of secondary users (SUs) in the same spectrum band, which is licensed to PUs $[3,4]$. The peak interference type of power control modeling for characterizing the underlay cognitive radio network (CRN) has been discussed in $[3,4,7]$. However, due to the highly dynamic nature of the CR network architecture, CR devices are extremely vulnerable to the mali-

K. Chopra is with the Department of Electrical Engineering, Indian Institute of Technology, Delhi, New Delhi, 110016 India e-mail: (see eez148071@ee.iitd.ac.in).

R. Bose is with the Department of Electrical Engineering, Indian Institute of Technology, Delhi, New Delhi, 110016 India e-mail: (see rbose@ee.iitd.ac.in).

A. Joshi is with the Department of Computer Science, University of Maryland, Baltimore County, Baltimore, MD 21250 USA e-mail: (see joshi@cs.umbc.edu). cious behavior [2,3]. Therefore, to ensure a reliable and secure data transmission, critical attention has been given in the literature to applications of the security issues in CRNs $[2-4,6,8,9]$. Information-theoretic security or physical layer security (PLS) has been developed as an effective secure wireless communications paradigm $[3,4,10-12]$, the theoretical foundation of which was laid by Wyner [13].

Diversity is an effective technique to combat the performance degradation in wireless communication systems caused due to fading. Cooperative diversity is incorporated in a multi-path fading environment with the help of relay nodes, to improve the communication reliability and throughput [10, 14, 15]. Maximal ratio combining (MRC) and selection combining (SC) are the diversity combining techniques, where the relayed signals as well as the signal from the source are combined to obtain the diversity gain [14]. Diversity reception scheme such as transmit antenna selection (TAS)/maximal ratio combining is discussed in [16] under high SNR regimes, for security enhancement in multiple-input multiple-output (MIMO) CRNs. High SNR analysis is carried out in [16], while, on the contrary, threshold-based relaying is considered in our work. Authors in [17] have studied secure switch-and-stay combining (SSSC) for two-phase underlay CRN. Unlike our study, direct links from the source to destination and eavesdropper are assumed to be in severe shadowing environments and thus non-existent in [17]. Authors in [18] have studied the secrecy performance of a half-duplex cognitive relay network. In [19], authors have considered physical-layer security protocols in multicast cognitive radio networks. Both in $[18,19]$, the direct link only between source and eavesdropper is taken into account, while the direct source to destination link is not available.

On the other hand, high SNR approximation is typically considered in the prior literature [16,20-25], where perfect decoding is assumed at each relay. However, for all the SNR regimes, this assumption might not be appropriate. Decoding error can occur in the system, as the signal strength might be degraded due to fading effect, such that the message is not correctly decoded by the relay. The secrecy performance of the cooperative system is thus affected by the quality of both source-relay and relaydestination link $[10,12,26]$. Whereas, high SNR assumption neglects the effect of the first hop link quality, which can actually be a bottleneck for secrecy performance in the cooperative communication $[10,12,26]$. To address the impact of sourcerelay link on the system secrecy in a better way, threshold-based relaying has been recently introduced, where, perfect decoding at the relay is only possible if the received signal-to-noise ratio (SNR) at the relay meets the particular threshold [7, 10,26]. Though the problems of secrecy in CRNs have been addressed by the researchers, the effect of threshold-based relaying and di- 
versity reception has yet not been well understood [27-30]. For mathematical simplicity, the combination of the relayed and the direct signal is not considered in the prior literature [17,27-30], by assuming that the direct link is in deep shadowing, which is practically not desirable in CRNs. This is because intuitively, due to the interference constraints from the primary user, the source power is random in nature, which indicates that the direct signal path might not be weak always, and therefore it should be considered [7,31]. Consequently, the QoS needs to be maintained by the relay in underlay CRNs, and it is thus employed even for short distance communication [31]. Recently, our works in [7], studied the secrecy performance of thresholdbased cognitive multi-relay system, with direct link only between secondary source and eavesdropper. The more practical scenario would be to include both source to eavesdropper and source to destination direct signal paths, such that two independent versions of the source message can be accessed to employ diversity combining techniques.

Our study is thus significantly different from the others discussed in the literature $[3,10,18,19,27-29,31-33]$, as it is motivated to reasonably examine the joint impact of the relayed and direct signal paths on cognitive system secrecy at both the destination and eavesdropper. We have hence presented a detailed and comprehensive secrecy analysis of employing both MRC and SC diversity receivers at the secondary destination and eavesdropper with all possible permutations, and have justified the best possible combination for secrecy of underlay cognitive relay system. It has been shown that how the direct signal paths have a significant impact on the cognitive system secrecy and the secrecy performance becomes more sensitive to the channel quality of source to eavesdropper than source to destination, especially at the higher secrecy rates. To further account for the effect of first hop link quality on system secrecy, threshold-based relaying has also been considered in this paper. We have organized the rest of our paper as follows. Section II describes the underlay cognitive relay threshold-based system model. The outage probability of the cognitive relay system with SC and MRC diversity schemes is evaluated in Section III. In Section IV, numerical results are discussed to support the analytical expressions. Section V concludes this study.

\section{System Model}

We consider a secondary source $S S$, that transmits its information to a secondary destination $S D$, through a single DF secondary relay $S R$, working in a dual-hop mode as shown in the Fig.1, in the presence of a secondary eavesdropper $S E$. In this underlay cognitive spectrum sharing, the transmission over the same spectrum by $S U$ and $P U$ are allowed, till there is no detection problem at the primary destination $P D$, due to the interference caused by the $S U[3,4,32]$. This constraint the powers of $S S$ and $S R$, such that the interference received at $P D$ should be lower than the maximum tolerable interference power level $I_{p}$. In addition, the maximum transmit power constraint is also considered, such that $S S$ and $S R$ powers are less than $P$, where $P$ is taken as the maximum transmit power $[3,4,7,32]$. Using both maximum transmit power and interference constraints, the $S S$ and $S R$ powers are limited as

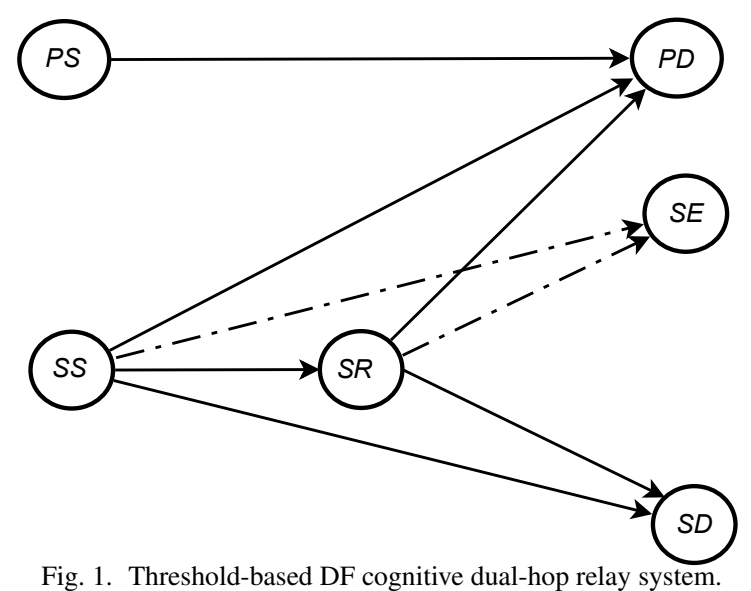

$P_{s} \leq \min \left(\frac{I_{p}}{\left|h_{s p}\right|^{2}}, P\right), P_{r} \leq \min \left(\frac{I_{p}}{\left|h_{r p}\right|^{2}}, P\right)$ respectively. For extremely large values of $P$, the transmit powers $P_{s}$ and $P_{r}$ of $S S$ and $S R$ are modeled as $P_{s}=\frac{I_{p}}{\left|h_{s p}\right|^{2}}, P_{r}=\frac{I_{p}}{\left|h_{r p}\right|^{2}}$. This peak interference type of power control modeling for characterizing the underlay cognitive network is assumed in our work, which is also widely used in prior literature $[3,4,7,32]$. We have not ignored the presence of direct $S S-S D$ and $S S-S E$ links in our cognitive system. The random nature of source powers due to interference constraints in the dual-hop CRN, indicates that the direct path signal might not be weak always, and therefore it should be considered [31]. The links between various nodes work in half-duplex mode in our system model and we have modeled them as Rayleigh flat fading mutually independent channels.

The SNR $\Gamma_{x y}$, between any two random nodes such as $x$ and $y[10,12]$, is given by

$$
\Gamma_{x y}=\frac{P_{x}\left|h_{x y}\right|^{2}}{N_{0_{y}}},
$$

where the transmit power is denoted by $P_{x}$ at the node $x$, the noise variance is given by $N_{0_{y}}$ at the node $y$ for additive white Gaussian noise (AWGN).$\Gamma_{x y}$ is exponentially distributed and $1 / \beta_{x y}$ is taken as the mean value, as $h_{x y}$ is Rayleigh distributed, [34]. It is given as $\Gamma_{x y} \sim \mathcal{E}\left(\beta_{\S \dagger}\right)$, where $\beta_{x y}$ is taken as the parameter of exponential distribution. The $S S-S R$ channels $h_{s r}$, $S R-S D$ channels $h_{r d}, S R-S E$ channels $h_{r e}, S S-P D$ channels $h_{s p}, S R-P D$ channels $h_{r p}, S S-S D$ channels $h_{s d}$, and $S S-S E$ channels $h_{s e}$, are slowly varying Rayleigh flat fading channels $[10,12]$. Without loss of generality, let $N_{s r}, N_{r d}$, $N_{r e}, N_{s p}, N_{r p}, N_{s d}$ and $N_{s e}$ denote the variances of AWGN for $S S-S R, S R-S D, S R-S E, S S-P D, S R-P D$, $S S-S D$ and $S S-S E$ links respectively. The SNRs $\Gamma_{s r}$, $\Gamma_{r d}, \Gamma_{r e}, \Gamma_{s p}, \Gamma_{r p}, \Gamma_{s d}$ and $\Gamma_{s e}$ are exponentially distributed which can be represented using (1), with link quality taken as $\gamma_{s r}=\frac{\left|h_{s r}\right|^{2}}{N_{s r}}, \gamma_{r d}=\frac{\left|h_{r d}\right|^{2}}{N_{r d}}, \gamma_{r e}=\frac{\left|h_{r e}\right|^{2}}{N_{r e}}, \gamma_{s d}=\frac{\left|h_{s d}\right|^{2}}{N_{s d}}$, $\gamma_{s e}=\frac{\left|h_{s e}\right|^{2}}{N_{s e}}, \gamma_{s p}=\left|h_{s p}\right|^{2}$ and $\gamma_{r p}=\left|h_{r p}\right|^{2}$, with average values $1 / \beta_{s r}, 1 / \beta_{r d}, 1 / \alpha_{r e}, 1 / \beta_{s d}, 1 / \alpha_{s e}, 1 / \theta_{s p}$ and $1 / \theta_{r p}$ respectively, where the parameters of exponential distribution are $\beta_{s r}, \beta_{r d}, \alpha_{r e}, \beta_{s d}, \alpha_{s e}, \theta_{s p}$ and $\theta_{r p}$.

When instantaneous secrecy rate of this cooperative relay system becomes lower than the required secrecy rate, given as $R_{s}$ 
where, $R_{s}>0$ and $\rho=2^{2 R_{s}}[10,35]$, an outage event occurs. $\rho$ is used for directly mapping $R_{s}$, and the outage probability $P_{o}$, is taken as the probability that this outage event has successfully occurred $[10,12]$. Without assuming correct decoding at the relay, here we take into consideration that the relay can perfectly decode and re-transmit the message, only if the received SNR meets the particular threshold SNR, taken as $\gamma_{t h}$ for the $S S-S R$ link [10,21].

Achievable secrecy rate is given as the difference between the information rate of main channel and eavesdropper channel $[10,12,13]$

$$
C_{s} \triangleq \frac{1}{2}\left[\log _{2}\left(\frac{1+\Gamma_{M}}{1+\Gamma_{E}}\right)\right]^{+},
$$

where $(x)^{+} \triangleq \max (0, x), \Gamma_{M}$ and $\Gamma_{E}$ are the respective SNRs at $S D$ and $S E$. Here, the $1 / 2$ term denotes that in order to finish the dual-hop transmission process, we require two time phase. The relay decodes the message transmitted by the source, in the first time phase. In the second time phase, the message is reencoded and forwarded by relay to the receiver.

For the random variable $Z$, which is exponentially distributed with parameter $\beta_{a b}$, the $\mathrm{CDF}$ is given as

$$
\begin{aligned}
F_{Z}(z) & =\mathbb{P}[Z \leq z] \\
& =1-e^{-z \beta_{a b}},
\end{aligned}
$$

and the corresponding PDF is obtained by differentiating (3) with respect to $z$ as

$$
f_{Z}(z)=\beta_{a b} e^{-z \beta_{a b}}
$$

For MRC, the random variable $Z$ is the sum of two random variables $A$ and $B$, i.e., $Z=A+B$ where $A$ and $B$ are exponentially distributed with parameters $\beta_{a b}$ and $\beta_{a^{\prime} b^{\prime}}$, the CDF is given as

$$
\begin{aligned}
F_{Z}(z) & =\mathbb{P}[A+B \leq z] \\
& =\mathbb{P}[A \leq z-B] \\
& =1-\frac{\beta_{a^{\prime} b^{\prime}} e^{-z \beta_{a b}}}{\beta_{a^{\prime} b^{\prime}}-\beta_{a b}}-\frac{\beta_{a b} e^{-z \beta_{a^{\prime} b^{\prime}}}}{\beta_{a b}-\beta_{a^{\prime} b^{\prime}}},
\end{aligned}
$$

and the corresponding PDF is obtained by differentiating (5) with respect to $z$ as

$$
f_{Z}(z)=\frac{\beta_{a^{\prime} b^{\prime}} \beta_{a b} e^{-z \beta_{a b}}}{\beta_{a^{\prime} b^{\prime}}-\beta_{a b}}+\frac{\beta_{a b} \beta_{a^{\prime} b^{\prime}} e^{-z \beta_{a^{\prime} b^{\prime}}}}{\beta_{a b}-\beta_{a^{\prime} b^{\prime}}} .
$$

For SC, the random variable $Z$ is the max of two random variables $A$ and $B$, i.e., $Z=\max (A, B)$ where $A$ and $B$ are exponentially distributed with parameters $\beta_{a b}$ and $\beta_{a^{\prime} b^{\prime}}$, the CDF is given as

$$
\begin{aligned}
F_{Z}(z) & =\mathbb{P}[\max (A, B) \leq z] \\
& =\mathbb{P}[A \leq z] \mathbb{P}[B \leq z] \\
& =1-e^{-z \beta_{a b}}-e^{-z \beta_{a^{\prime} b^{\prime}}}+e^{-z\left(\beta_{a b}+\beta_{a^{\prime} b^{\prime}}\right)},
\end{aligned}
$$

and the corresponding PDF is obtained by differentiating (7) with respect to $z$ as

$$
\begin{aligned}
f_{Z}(z)= & \beta_{a b} e^{-z \beta_{a b}}+\beta_{a^{\prime} b^{\prime}} e^{-z \beta_{a^{\prime} b^{\prime}}} \\
& -\left(\beta_{a b}+\beta_{a^{\prime} b^{\prime}}\right) e^{-z\left(\beta_{a b}+\beta_{a^{\prime} b^{\prime}}\right)} .
\end{aligned}
$$

We have investigated four scenarios in our study. First is when SC diversity scheme is employed at both $S D$ and $S E$, such that when $\Gamma_{s r} \geq \gamma_{t h}$, the relay correctly decodes the message and $\Gamma_{M}^{S C}=\max \left(\Gamma_{r d}, \Gamma_{s d}\right)$ is the SNR at $S D$ and $\Gamma_{E}^{S C}=$ $\max \left(\Gamma_{r e}, \Gamma_{s e}\right)$ is the SNR at $S E$. The second is when SC diversity scheme is employed at $S D$ and MRC diversity scheme is employed at $S E$, such that when $\Gamma_{s r} \geq \gamma_{t h}$, the relay correctly decodes the message and $\Gamma_{M}^{S C}=\max \left(\Gamma_{r d}, \Gamma_{s d}\right)$ is the SNR at $S D$ and $\Gamma_{E}^{M R C}=\Gamma_{r e}+\Gamma_{s e}$ is the SNR at $S E$. The third is when MRC diversity scheme is employed at $S D$ and SC diversity scheme is employed at $S E$, such that when $\Gamma_{s r} \geq \gamma_{t h}$, the relay correctly decodes the message and $\Gamma_{M}^{M R C}=\Gamma_{r d}+\Gamma_{s d}$ is the SNR at $S D$ and $\Gamma_{E}^{S C}=\max \left(\Gamma_{r e}, \Gamma_{s e}\right)$ is the SNR at $S E$. The fourth is when MRC diversity scheme is employed at both $S D$ and $S E$, such that when $\Gamma_{s r} \geq \gamma_{t h}$, the relay correctly decodes the message and $\Gamma_{M}^{M R C}=\bar{\Gamma}_{r d}+\Gamma_{s d}$ is the SNR at $S D$ and $\Gamma_{E}^{M R C}=\Gamma_{r e}+\Gamma_{s e}$ is the SNR at $S E$. When $\Gamma_{s r}<\gamma_{t h}$, the relay does not transmit at all, thus only direct $S S-S E$ and $S S-S D$ communication link exists, where $\Gamma_{M}=\Gamma_{s d}$ is taken as the main link SNR at $S D$ and $\Gamma_{E}=\Gamma_{s e}$ is taken as the eavesdropper link SNR at $S E$ for all the four scenarios.

\section{Secrecy Outage Probability of Cognitive Single Relay System}

This section discusses the $P_{o}$ for the cognitive thresholdbased relay system where, both the direct signal paths between $S S-S D$ and $S S-S E$ exists. The outage probability has been evaluated in this section by finding out the conditional outage probability of cognitive system, when the relay could successfully decodes the source message and when it could not. $\mathbb{E}[\cdot]$ is taken as the expectation operator, which involves the averaging over random variables $\gamma_{s p}$ and $\gamma_{r p}$. Hence, we have followed the law of total probability for obtaining the outage probability for the four scenarios, and it is given as

$$
\begin{aligned}
P_{o} & =\mathbb{E}_{\gamma_{r p}}\left[\mathbb { E } _ { \gamma _ { s p } } \left[\mathbb{P}\left[C_{s}<R_{s} \mid \Gamma_{s r} \geq \gamma_{t h}, \gamma_{s p}, \gamma_{r p}\right] \times\right.\right. \\
& \mathbb{P}\left[\Gamma_{s r} \geq \gamma_{t h} \mid \gamma_{s p}, \gamma_{r p}\right]+\mathbb{P}\left[C_{s}<R_{s} \mid \Gamma_{s r}<\gamma_{t h}, \gamma_{s p}, \gamma_{r p}\right] \\
& \left.\left.\times \mathbb{P}\left[\Gamma_{s r}<\gamma_{t h} \mid \gamma_{s p}, \gamma_{r p}\right]\right]\right]
\end{aligned}
$$

A. Secrecy Outage With SC at both Destination and Eavesdrop$\operatorname{per}(S C-S C)$

The $P_{o}$ for the first scenario is given as

$$
\begin{aligned}
P_{o} & =\mathbb{E}\left[\mathbb{P}\left[\frac{1+\max \left(\frac{I_{p} \gamma_{r d}}{\gamma_{r p}}, \frac{I_{p} \gamma_{s d}}{\gamma_{s p}}\right)}{1+\max \left(\frac{I_{p} \gamma_{r e}}{\gamma_{r p}}, \frac{I_{p} \gamma_{s e}}{\gamma_{s p}}\right)}<\rho \mid \Gamma_{s r} \geq \gamma_{t h}, \gamma_{s p}, \gamma_{r p}\right]\right. \\
& \times\left[1-\mathbb{P}\left[\frac{I_{p} \gamma_{s r}}{\gamma_{s p}}<\gamma_{t h} \mid \gamma_{s p}, \gamma_{r p}\right]\right]
\end{aligned}
$$




$$
\begin{aligned}
& +\mathbb{P}\left[\frac{1+\frac{I_{p} \gamma_{s d}}{\gamma_{s p}}}{1+\frac{I_{p} \gamma_{s e}}{\gamma_{s p}}}<\rho \mid \Gamma_{s r}<\gamma_{t h}, \gamma_{s p}, \gamma_{r p}\right] \\
& \left.\times \mathbb{P}\left[\frac{I_{p} \gamma_{s r}}{\gamma_{s p}}<\gamma_{t h} \mid \gamma_{s p}, \gamma_{r p}\right]\right]
\end{aligned}
$$

Expressions for the terms $Q_{1}$ to $Q_{11}$ in (11) are presented in the Appendix, after using some algebraic simplifications and performing partial fractions.

\section{B. Secrecy Outage With SC at Destination and MRC at Eaves- dropper (SC-MRC)}

The $P_{o}$ for the second scenario is given as

$$
\begin{aligned}
P_{o} & =\mathbb{E}\left[\mathbb{P}\left[\frac{1+\max \left(\frac{I_{p} \gamma_{r d}}{\gamma_{r p}}, \frac{I_{p} \gamma_{s d}}{\gamma_{s p}}\right)}{1+\left(\frac{I_{p} \gamma_{r e}}{\gamma_{r p}}+\frac{I_{p} \gamma_{s e}}{\gamma_{s p}}\right)}<\rho \mid \Gamma_{s r} \geq \gamma_{t h}, \gamma_{s p}, \gamma_{r p}\right]\right. \\
& \times\left[1-\mathbb{P}\left[\frac{I_{p} \gamma_{s r}}{\gamma_{s p}}<\gamma_{t h} \mid \gamma_{s p}, \gamma_{r p}\right]\right] \\
& +\mathbb{P}\left[\frac{1+\frac{I_{p} \gamma_{s d}}{\gamma_{s p}}}{1+\frac{I_{p} \gamma_{s e}}{\gamma_{s p}}}<\rho \mid \Gamma_{s r}<\gamma_{t h}, \gamma_{s p}, \gamma_{r p}\right] \\
& \left.\times \mathbb{P}\left[\frac{I_{p} \gamma_{s r}}{\gamma_{s p}}<\gamma_{t h} \mid \gamma_{s p}, \gamma_{r p}\right]\right]
\end{aligned}
$$

Expressions for the terms $R_{1}$ to $R_{8}$ in (13) are presented in the Appendix, after using some algebraic simplifications and performing partial fractions.

\section{Secrecy Outage With MRC at Destination and SC at Eaves- dropper (MRC-SC)}

The $P_{o}$ for the third scenario is given as

$$
\begin{aligned}
P_{o} & =\mathbb{E}\left[\mathbb{P}\left[\frac{1+\left(\frac{I_{p} \gamma_{r d}}{\gamma_{r p}}+\frac{I_{p} \gamma_{s d}}{\gamma_{s p}}\right)}{1+\max \left(\frac{I_{p} \gamma_{r e}}{\gamma_{r p}}, \frac{I_{p} \gamma_{s e}}{\gamma_{s p}}\right)}<\rho \mid \Gamma_{s r} \geq \gamma_{t h}, \gamma_{s p}, \gamma_{r p}\right]\right. \\
& \times\left[1-\mathbb{P}\left[\frac{I_{p} \gamma_{s r}}{\gamma_{s p}}<\gamma_{t h} \mid \gamma_{s p}, \gamma_{r p}\right]\right] \\
& +\mathbb{P}\left[\frac{1+\frac{I_{p} \gamma_{s d}}{\gamma_{s p}}}{1+\frac{I_{p} \gamma_{s e}}{\gamma_{s p}}}<\rho \mid \Gamma_{s r}<\gamma_{t h}, \gamma_{s p}, \gamma_{r p}\right] \\
& \left.\times \mathbb{P}\left[\frac{I_{p} \gamma_{s r}}{\gamma_{s p}}<\gamma_{t h} \mid \gamma_{s p}, \gamma_{r p}\right]\right]
\end{aligned}
$$

Expressions for the terms $S_{1}$ to $S_{8}$ in (15) are presented in the Appendix, after using some algebraic simplifications and performing partial fractions.

\section{Secrecy Outage With MRC at both Destination and Eaves- dropper (MRC-MRC)}

The $P_{o}$ for the fourth scenario is given as

$$
\begin{aligned}
P_{o} & =\mathbb{E}\left[\mathbb{P}\left[\frac{1+\left(\frac{I_{p} \gamma_{r d}}{\gamma_{r p}}+\frac{I_{p} \gamma_{s d}}{\gamma_{s p}}\right)}{1+\left(\frac{I_{p} \gamma_{r e}}{\gamma_{r p}}+\frac{I_{p} \gamma_{s e}}{\gamma_{s p}}\right)}<\rho \mid \Gamma_{s r} \geq \gamma_{t h}, \gamma_{s p}, \gamma_{r p}\right]\right. \\
& \times\left[1-\mathbb{P}\left[\frac{I_{p} \gamma_{s r}}{\gamma_{s p}}<\gamma_{t h} \mid \gamma_{s p}, \gamma_{r p}\right]\right] \\
& +\mathbb{P}\left[\frac{1+\frac{I_{p} \gamma_{s d}}{\gamma_{s p}}}{1+\frac{I_{p} \gamma_{s e}}{\gamma_{s p}}}<\rho \mid \Gamma_{s r}<\gamma_{t h}, \gamma_{s p}, \gamma_{r p}\right] \\
& \left.\times \mathbb{P}\left[\frac{I_{p} \gamma_{s r}}{\gamma_{s p}}<\gamma_{t h} \mid \gamma_{s p}, \gamma_{r p}\right]\right]
\end{aligned}
$$

Expressions for the terms $T_{1}$ to $T_{6}$ in (17) are presented in the Appendix, after using some algebraic simplifications and performing partial fractions. Using these, $P_{o}$ for all four cases is evaluated and the secrecy performance analysis is done further in the numerical section.

\section{E. Secrecy Outage With No Direct Link}

This section discusses secrecy performance of the cognitive relay system, with an assumption that both the direct signal paths $S S-S D$ and $S S-S E$ are not present due to deep shadow fading [7]. The $P_{o}$ is given as

$$
\begin{aligned}
P_{o}= & \mathbb{E}\left[\mathbb{P}\left[\frac{1}{2}\left[\log _{2}\left(\frac{1+\Gamma_{r d}}{1+\Gamma_{r e}}\right)\right]<R_{s} \mid \Gamma_{s r} \geq \gamma_{t h}, \gamma_{s p}, \gamma_{r p}\right]\right. \\
\times & \left.\mathbb{P}\left[\Gamma_{s r} \geq \gamma_{t h} \mid \gamma_{s p}, \gamma_{r p}\right]+\mathbb{P}\left[\Gamma_{s r}<\gamma_{t h} \mid \gamma_{s p}, \gamma_{r p}\right]\right] \\
= & \int_{0}^{+\infty} \int_{0}^{+\infty}\left[\left(1-\frac{\alpha_{r e} e^{-\left(\frac{\rho-1}{I_{p}}\right) \beta_{r d} y}}{\rho \beta_{r d}+\alpha_{r e}}\right) e^{-\frac{\gamma_{t h} \beta_{s r x}}{I_{p}}}\right. \\
& \left.+\left(1-e^{-\frac{\gamma_{t h} \beta_{s r} x}{I_{p}}}\right)\right] \theta_{s p} e^{-\theta_{s p} x} \theta_{r p} e^{-\theta_{r p} y} d x d y
\end{aligned}
$$

The closed-form expression of the $P_{o}$ for cognitive relay system from (18) is obtained as

$P_{o}=\left(1-\frac{\alpha_{r e} \theta_{r p} \theta_{s p}}{\left(\rho \beta_{r d}+\alpha_{r e}\right)\left(\theta_{r p}+\left(\frac{\rho-1}{I_{p}}\right) \beta_{r d}\right)\left(\theta_{s p}+\frac{\gamma_{t h} \beta_{s p}}{I_{p}}\right)}\right)$

\section{F. Asymptotic Analysis}

For the asymptotic behavior analysis, when $1 / \beta_{s r}=$ $1 / \beta_{r d}=1 / \beta \rightarrow \infty$, the $P_{o}$ for cognitive threshold-based single relay system in (19) is given as

$$
P_{o}=\frac{\beta_{r d}\left(\rho \theta_{r p}+\frac{\alpha_{r e}(\rho-1)}{I_{p}}\right)}{\alpha_{r e} \theta_{r p}}+\frac{\gamma_{t h} \beta_{s r}}{I_{p} \theta_{s p}}
$$




$$
\begin{aligned}
& P_{o}=\underbrace{\mathbb{E}\left[e^{-\frac{\gamma_{t h} \beta_{s r} \gamma_{s p}}{I_{p}}}\right]}_{Q_{1}}-\underbrace{\mathbb{E}\left[\frac{e^{-\frac{\gamma_{t h} \beta_{s r} \gamma_{s p}}{I_{p}}} \gamma_{r p} \alpha_{r e} e^{-\left(\frac{\rho-1}{I_{p}}\right) \beta_{s d} \gamma_{s p}}}{\left(\rho \beta_{s d} \gamma_{s p}+\alpha_{r e} \gamma_{r p}\right)}\right]}_{Q_{2}} \\
& \underbrace{\mathbb{E}\left[\frac{e^{-\frac{\gamma_{t h} \beta_{s r} \gamma_{s p}}{I_{p}}} \gamma_{s p} \alpha_{s e} e^{-\left(\frac{\rho-1}{I_{p}}\right) \beta_{s d} \gamma_{s p}}}{\left(\rho \beta_{s d} \gamma_{s p}+\alpha_{s e} \gamma_{s p}\right)}\right]}_{Q_{3}}+\underbrace{\mathbb{E}\left[\frac{e^{-\frac{\gamma_{t h} \beta_{s r} \gamma_{s p}}{I_{p}}}\left(\gamma_{s p} \alpha_{s e}+\alpha_{r e} \gamma_{r p}\right) e^{-\left(\frac{\rho-1}{I_{p}}\right) \beta_{s d} \gamma_{s p}}}{\left(\rho \beta_{s d} \gamma_{s p}+\alpha_{s e} \gamma_{s p}+\alpha_{r e} \gamma_{r p}\right)}\right]}_{Q_{4}} \\
& -\underbrace{\mathbb{E}\left[\frac{e^{-\frac{\gamma_{t h} \beta_{s r} \gamma_{s p}}{I_{p}}} \gamma_{r p} \alpha_{r e} e^{-\left(\frac{\rho-1}{I_{p}}\right) \beta_{r d} \gamma_{r p}}}{\left(\rho \beta_{r d} \gamma_{r p}+\alpha_{r e} \gamma_{r p}\right)}\right]}_{Q_{5}}-\underbrace{\mathbb{E}\left[\frac{e^{-\frac{\gamma_{t h} \beta_{s r} \gamma_{s p}}{I_{p}}} \gamma_{s p} \alpha_{s e} e^{-\left(\frac{\rho-1}{I_{p}}\right) \beta_{r d} \gamma_{r p}}}{\left(\rho \beta_{r d} \gamma_{r p}+\alpha_{s e} \gamma_{s p}\right)}\right]}_{Q_{6}} \\
& +\underbrace{\mathbb{E}\left[\frac{e^{-\frac{\gamma_{t h} \beta_{s r} \gamma_{s p}}{I_{p}}}\left(\gamma_{s p} \alpha_{s e}+\alpha_{r e} \gamma_{r p}\right) e^{-\left(\frac{\rho-1}{I_{p}}\right) \beta_{r d} \gamma_{r p}}}{\left(\rho \beta_{r d} \gamma_{r p}+\alpha_{s e} \gamma_{s p}+\alpha_{r e} \gamma_{r p}\right)}\right]}_{Q_{7}}+\underbrace{\mathbb{E}\left[\frac{e^{-\frac{\gamma_{t h} \beta_{s r} \gamma_{s p}}{I_{p}}} \gamma_{r p} \alpha_{r e} e^{-\left(\frac{\rho-1}{I_{p}}\right)\left(\beta_{s d} \gamma_{s p}+\beta_{r d} \gamma_{r p}\right)}}{\left(\rho\left(\beta_{s d} \gamma_{s p}+\beta_{r d} \gamma_{r p}\right)+\alpha_{r e} \gamma_{r p}\right)}\right]}_{Q_{8}} \\
& +\underbrace{\mathbb{E}\left[\frac{e^{-\frac{\gamma_{t h} \beta_{s r} \gamma_{s p}}{I_{p}}} \gamma_{s p} \alpha_{s e} e^{-\left(\frac{\rho-1}{I_{p}}\right)\left(\beta_{s d} \gamma_{s p}+\beta_{r d} \gamma_{r p}\right)}}{\left(\rho\left(\beta_{s d} \gamma_{s p}+\beta_{r d} \gamma_{r p}\right)+\alpha_{s e} \gamma_{s p}\right)}\right]}_{Q_{9}}-\underbrace{\mathbb{E}\left[\frac{e^{-\frac{\gamma_{t h} \beta_{s r} \gamma_{s p}}{I_{p}}}\left(\gamma_{s p} \alpha_{s e}+\alpha_{r e} \gamma_{r p}\right) e^{-\left(\frac{\rho-1}{I_{p}}\right)\left(\beta_{s d} \gamma_{s p}+\beta_{r d} \gamma_{r p}\right)}}{\left(\rho\left(\beta_{s d} \gamma_{s p}+\beta_{r d} \gamma_{r p}\right)+\alpha_{s e} \gamma_{s p}+\alpha_{r e} \gamma_{r p}\right)}\right]}_{Q_{10}} \\
& +\underbrace{\mathbb{E}\left[\left(1-\frac{\alpha_{s e} e^{-\left(\frac{\rho-1}{I_{p}}\right) \beta_{s d} \gamma_{s p}}}{\rho \beta_{s d}+\alpha_{s e}}\right)\left(1-e^{\left.-\frac{\gamma_{t h} \beta_{s r} \gamma_{s p}}{I_{p}}\right)}\right)\right]}_{Q_{11}}
\end{aligned}
$$

$$
\begin{aligned}
& P_{o}=\underbrace{\mathbb{E}\left[e^{-\frac{\gamma_{t h} \beta_{s r} \gamma_{s p}}{I_{p}}}\right]}_{R_{1}}-\underbrace{\mathbb{E}\left[\frac{e^{-\frac{\gamma_{t h} \beta_{s r} \gamma_{s p}}{I_{p}}} \gamma_{r p} \alpha_{r e} \alpha_{s e} \gamma_{s p} e^{-\left(\frac{\rho-1}{I_{p}}\right) \beta_{s d} \gamma_{s p}}}{\left(\rho \beta_{s d} \gamma_{s p}+\alpha_{s e} \gamma_{s p}\right)\left(\alpha_{r e} \gamma_{r p}-\alpha_{s e} \gamma_{s p}\right)}\right]}_{R_{2}} \\
& -\underbrace{\mathbb{E}\left[\frac{e^{-\frac{\gamma_{t h} \beta_{s r} \gamma_{s p}}{I_{p}}} \gamma_{r p} \alpha_{r e} \alpha_{s e} \gamma_{s p} e^{-\left(\frac{\rho-1}{I_{p}}\right) \beta_{s d} \gamma_{s p}}}{\left(\rho \beta_{s d} \gamma_{s p}+\alpha_{r e} \gamma_{r p}\right)\left(\alpha_{s e} \gamma_{s p}-\alpha_{r e} \gamma_{r p}\right)}\right]}_{R_{3}}-\underbrace{\mathbb{E}\left[\frac{e^{-\frac{\gamma_{t h} \beta_{s r} \gamma_{s p}}{I_{p}}} \gamma_{r p} \alpha_{r e} \alpha_{s e} \gamma_{s p} e^{-\left(\frac{\rho-1}{I_{p}}\right) \beta_{r d} \gamma_{r p}}}{\left(\rho \beta_{r d} \gamma_{r p}+\alpha_{s e} \gamma_{s p}\right)\left(\alpha_{r e} \gamma_{r p}-\alpha_{s e} \gamma_{s p}\right)}\right]}_{R_{4}} \\
& -\underbrace{\mathbb{E}\left[\frac{e^{-\frac{\gamma_{t h} \beta_{s r} \gamma_{s p}}{I_{p}}} \gamma_{r p} \alpha_{r e} \alpha_{s e} \gamma_{s p} e^{-\left(\frac{\rho-1}{I_{p}}\right) \beta_{r d} \gamma_{r p}}}{\left(\rho \beta_{r d} \gamma_{r p}+\alpha_{r e} \gamma_{r p}\right)\left(\alpha_{s e} \gamma_{s p}-\alpha_{r e} \gamma_{r p}\right)}\right]}_{R_{5}}+\underbrace{\mathbb{E}\left[\frac{e^{-\frac{\gamma_{t h} \beta_{s r} \gamma_{s p}}{I_{p}}} \gamma_{r p} \alpha_{r e} \alpha_{s e} \gamma_{s p} e^{-\left(\frac{\rho-1}{I_{p}}\right)\left(\beta_{s d} \gamma_{s p}+\beta_{r d} \gamma_{r p}\right)}}{\left(\rho\left(\beta_{s d} \gamma_{s p}+\beta_{r d} \gamma_{r p}\right)+\alpha_{s e} \gamma_{s p}\right)\left(\alpha_{r e} \gamma_{r p}-\alpha_{s e} \gamma_{s p}\right)}\right]}_{R_{6}} \\
& +\underbrace{\mathbb{E}\left[\frac{e^{-\frac{\gamma_{t h} \beta_{s r} \gamma_{s p}}{I_{p}}} \gamma_{r p} \alpha_{r e} \alpha_{s e} \gamma_{s p} e^{-\left(\frac{\rho-1}{I_{p}}\right)\left(\beta_{s d} \gamma_{s p}+\beta_{r d} \gamma_{r p}\right)}}{\left(\rho\left(\beta_{s d} \gamma_{s p}+\beta_{r d} \gamma_{r p}\right)+\alpha_{r e} \gamma_{r p}\right)\left(\alpha_{s e} \gamma_{s p}-\alpha_{r e} \gamma_{r p}\right)}\right]}_{R_{7}}+\underbrace{\mathbb{E}\left[\left(1-\frac{\alpha_{s e} e^{-\left(\frac{\rho-1}{I_{p}}\right) \beta_{s d} \gamma_{s p}}}{\rho \beta_{s d}+\alpha_{s e}}\right)\left(1-e^{\left.-\frac{\gamma_{t h} \beta_{s r} \gamma_{s p}}{I_{p}}\right)}\right]\right.}_{R_{8}}
\end{aligned}
$$

$$
=\frac{1}{\frac{1}{\beta}}\left[\frac{\rho}{\alpha_{r e}}+\frac{(\rho-1)}{I_{p} \theta_{r p}}+\frac{\gamma_{t h}}{I_{p} \theta_{s p}}\right]
$$

We can observe from (20) that $P_{o}$ is inversely proportional to $1 / \beta$, and when the main channel SNR $=1 / \beta$ tends to infinity, $P_{o}$ tends to zero. $P_{o}$ as shown in (20) is directly proportional to $\gamma_{t h}, 1 / \alpha_{r e}$ and $\rho$. We can see that $P_{o}$ also decreases with an increase of $I_{p}$. Also, the diversity order, $D[10,12]$ is given by the power of $1 / \beta$ in the denominator of (20). As we have not considered any relay selection, it is intuitive that $D=1$, for this single relay cognitive system.

\section{Numerical Analysis}

This section presents the simulation results to validate the analytical expressions. All the secondary and primary nodes are assumed to have the same noise power. 


$$
\begin{aligned}
& P_{o}=\underbrace{\mathbb{E}\left[e^{-\frac{\gamma_{t h} \beta_{s r} \gamma_{s p}}{I_{p}}}\right]}_{S_{1}}-\underbrace{\mathbb{E}\left[\frac{e^{-\frac{\gamma_{t h} \beta_{s r} \gamma_{s p}}{I_{p}}} \gamma_{s p} \beta_{s d} \alpha_{s e} \gamma_{s p} e^{-\left(\frac{\rho-1}{I_{p}}\right) \beta_{r d} \gamma_{r p}}}{\left(\rho \beta_{r d} \gamma_{r p}+\alpha_{s e} \gamma_{s p}\right)\left(\beta_{s d} \gamma_{s p}-\beta_{r d} \gamma_{r p}\right)}\right]}_{S_{2}} \\
& \underbrace{-\left[\frac{e^{-\frac{\gamma_{t h} \beta_{s r} \gamma_{s p}}{I_{p}}} \gamma_{r p} \beta_{r d} \alpha_{s e} \gamma_{s p} e^{-\left(\frac{\rho-1}{I_{p}}\right) \beta_{s d} \gamma_{s p}}}{\left(\rho \beta_{s d} \gamma_{s p}+\alpha_{s e} \gamma_{s p}\right)\left(\beta_{r d} \gamma_{r p}-\beta_{s d} \gamma_{s p}\right)}\right]}_{S_{3}}-\underbrace{\mathbb{E}\left[\frac{e^{-\frac{\gamma_{t h} \beta_{s r} \gamma_{s p}}{I_{p}}} \gamma_{s p} \beta_{s d} \alpha_{r e} \gamma_{r p} e^{-\left(\frac{\rho-1}{I_{p}}\right) \beta_{r d} \gamma_{r p}}}{\left(\rho \beta_{r d} \gamma_{r p}+\alpha_{r e} \gamma_{r p}\right)\left(\beta_{s d} \gamma_{s p}-\beta_{r d} \gamma_{r p}\right)}\right]}_{S_{4}} \\
& -\underbrace{\mathbb{E}\left[\frac{e^{-\frac{\gamma_{t h} \beta_{s r} \gamma_{s p}}{I_{p}}} \gamma_{r p} \beta_{r d} \alpha_{r e} \gamma_{r p} e^{-\left(\frac{\rho-1}{I_{p}}\right) \beta_{s d} \gamma_{s p}}}{\left(\rho \beta_{s d} \gamma_{s p}+\alpha_{r e} \gamma_{r p}\right)\left(\beta_{r d} \gamma_{r p}-\beta_{s d} \gamma_{s p}\right)}\right]}_{S_{5}}+\underbrace{\mathbb{E}\left[\frac{e^{-\frac{\gamma_{t h} \beta_{s r} \gamma_{s p}}{I_{p}}} \gamma_{s p} \beta_{s d}\left(\alpha_{r e} \gamma_{r p}+\alpha_{s e} \gamma_{s p}\right) e^{-\left(\frac{\rho-1}{I_{p}}\right) \beta_{r d} \gamma_{r p}}}{\left(\rho \beta_{r d} \gamma_{r p}+\alpha_{s e} \gamma_{s p}+\alpha_{r e} \gamma_{r p}\right)\left(\beta_{s d} \gamma_{s p}-\beta_{r d} \gamma_{r p}\right)}\right]}_{S_{6}} \\
& +\underbrace{\mathbb{E}\left[\frac{e^{-\frac{\gamma_{t h} \beta_{s r} \gamma_{s p}}{I_{p}}} \gamma_{r p} \beta_{r d}\left(\alpha_{r e} \gamma_{r p}+\alpha_{s e} \gamma_{s p}\right) e^{-\left(\frac{\rho-1}{I_{p}}\right) \beta_{s d} \gamma_{s p}}}{\left(\rho \beta_{s d} \gamma_{s p}+\alpha_{s e} \gamma_{s p}+\alpha_{r e} \gamma_{r p}\right)\left(\beta_{r d} \gamma_{r p}-\beta_{s d} \gamma_{s p}\right)}\right]}_{S_{7}}+\underbrace{\mathbb{E}\left[( 1 - \frac { \alpha _ { s e } e ^ { - ( \frac { \rho - 1 } { I _ { p } } ) \beta _ { s d } \gamma _ { s p } } } { \rho \beta _ { s d } + \alpha _ { s e } } ) \left(1-e^{\left.\left.-\frac{\gamma_{t h} \beta_{s r} \gamma_{s p}}{I_{p}}\right)\right]}\right.\right.}_{S_{8}}
\end{aligned}
$$

$$
\begin{aligned}
& P_{o}=\underbrace{\mathbb{E}\left[e^{-\frac{\gamma_{t h} \beta_{s r} \gamma_{s p}}{I_{p}}}\right]}_{T_{1}}-\underbrace{\mathbb{E}\left[\frac{e^{-\frac{\gamma_{t h} \beta_{s r} \gamma_{s p}}{I_{p}}} \gamma_{s p} \beta_{s d} \alpha_{r e} \alpha_{s e} \gamma_{s p} e^{-\left(\frac{\rho-1}{I_{p}}\right) \beta_{r d} \gamma_{r p}}}{\left(\beta_{s d} \gamma_{s p}-\beta_{r d} \gamma_{r p}\right)\left(\alpha_{s e} \gamma_{s p}-\alpha_{r e} \gamma_{r p}\right)\left(\rho \beta_{r d}+\alpha_{r e}\right)}\right]}_{T_{2}} \\
& -\underbrace{\mathbb{E}\left[\frac{e^{-\frac{\gamma_{t h} \beta_{s r} \gamma_{s p}}{I_{p}}} \gamma_{s p} \gamma_{r p} \beta_{r d} \alpha_{r e} \alpha_{s e} \gamma_{r p} e^{-\left(\frac{\rho-1}{I_{p}}\right) \beta_{s d} \gamma_{s p}}}{\left(\beta_{r d} \gamma_{r p}-\beta_{s d} \gamma_{s p}\right)\left(\alpha_{s e} \gamma_{s p}-\alpha_{r e} \gamma_{r p}\right)\left(\rho \beta_{s d} \gamma_{s p}+\alpha_{r e} \gamma_{r p}\right)}\right]}_{T_{3}} \\
& -\underbrace{\mathbb{E}\left[\frac{e^{-\frac{\gamma_{t h} \beta_{s r} \gamma_{s p}}{I_{p}}} \gamma_{s p} \gamma_{r p} \beta_{s d} \alpha_{r e} \alpha_{s e} \gamma_{s p} e^{-\left(\frac{\rho-1}{I_{p}}\right) \beta_{r d} \gamma_{r p}}}{\left(\beta_{s d} \gamma_{s p}-\beta_{r d} \gamma_{r p}\right)\left(\alpha_{r e} \gamma_{r p}-\alpha_{s e} \gamma_{s p}\right)\left(\rho \beta_{r d} \gamma_{r p}+\alpha_{s e} \gamma_{s p}\right)}\right]}_{T_{4}} \\
& -\underbrace{\mathbb{E}\left[\frac{e^{-\frac{\gamma_{t h} \beta_{s r} \gamma_{s p}}{I_{p}}} \gamma_{r p} \beta_{r d} \alpha_{r e} \alpha_{s e} \gamma_{r p} e^{-\left(\frac{\rho-1}{I_{p}}\right) \beta_{s d} \gamma_{s p}}}{\left(\beta_{r d} \gamma_{r p}-\beta_{s d} \gamma_{s p}\right)\left(\alpha_{r e} \gamma_{r p}-\alpha_{s e} \gamma_{s p}\right)\left(\rho \beta_{s d}+\alpha_{s e}\right)}\right]}_{T_{5}} \\
& +\underbrace{\mathbb{E}\left[\left(1-\frac{\alpha_{s e} e^{-\left(\frac{\rho-1}{I_{p}}\right) \beta_{s d} \gamma_{s p}}}{\rho \beta_{s d}+\alpha_{s e}}\right)\left(1-e^{\left.-\frac{\gamma_{t h} \beta_{s r} \gamma_{s p}}{I_{p}}\right)}\right)\right]}_{T_{6}}
\end{aligned}
$$

Fig. 2 shows the $P_{o}$ for cognitive system under SC-SC scenario as given in (11), with total SNR $1 / \beta$. The figure is plotted with different $R_{s}=0.1,2.0,1 / \beta_{s d}=6,9 \mathrm{~dB}, 1 / \alpha_{s e}=6,9 \mathrm{~dB}$ and fixed $\gamma_{t h}=3 \mathrm{~dB}, 1 / \alpha_{r e}=1 / \alpha=9 \mathrm{~dB}, I_{p}=9 \mathrm{~dB}$, $1 / \theta_{s p}=3 \mathrm{~dB}, 1 / \theta_{r p}=3 \mathrm{~dB}$. It is interesting to observe from the plot that the secrecy performance is improved by increasing $1 / \beta_{s d}$ from $6 \mathrm{~dB}$ to $9 \mathrm{~dB}$ for a given $1 / \alpha_{s e}=3 \mathrm{~dB}$ at lower secrecy rates, while, at higher secrecy rates there is no significant improvement. However, the secrecy performance significantly decreases by increasing $1 / \alpha_{\text {se }}$ from $6 \mathrm{~dB}$ to $9 \mathrm{~dB}$ for a given $1 / \beta_{s d}=3 \mathrm{~dB}$ both at high and low secrecy rates. The secrecy outage is thus more sensitive to changes in the quality of di- rect link between $S S-S E$ than $S S-S D$, especially at the higher secrecy rates. This makes the direct link between secondary source to eavesdropper more crucial at higher secrecy rates for secure communication.

Fig. 3 shows the comparison of $P_{o}$ for cognitive system, as given in (11), (13), (15) and (17), with total SNR $1 / \beta$. The figure is plotted with different $R_{s}=0.1,1.0$ and fixed $\gamma_{t h}=3 \mathrm{~dB}$, $1 / \alpha_{r e}=1 / \alpha=9 \mathrm{~dB}, I_{p}=9 \mathrm{~dB}, 1 / \beta_{s d}=3 \mathrm{~dB}, 1 / \alpha_{s e}=3$ $\mathrm{dB}, 1 / \theta_{s p}=3 \mathrm{~dB}, 1 / \theta_{r p}=3 \mathrm{~dB}$.It is observed from the figure that secrecy performance of the cognitive relay system is best when diversity scheme MRC is used at $S D$ and SC is used at $S E$. The performance is worst when diversity scheme $\mathrm{SC}$ is 


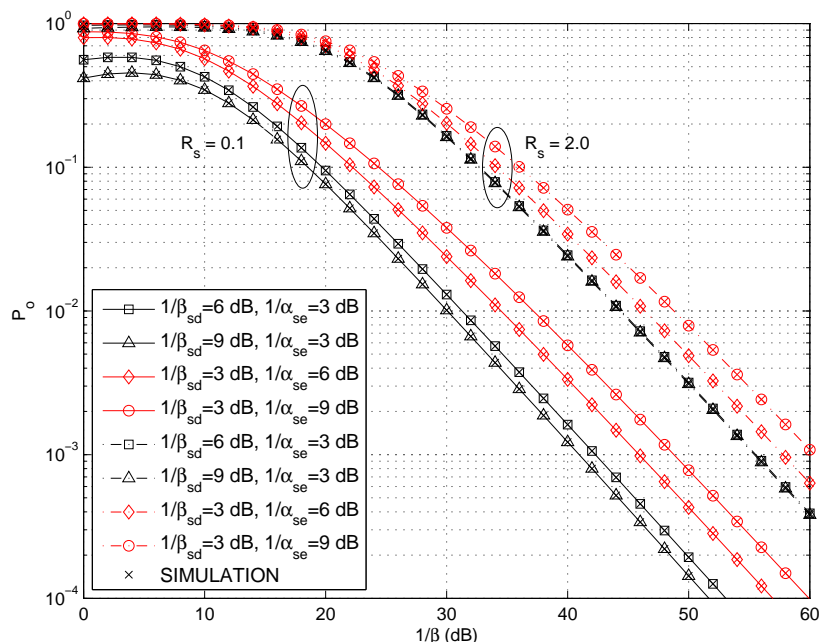

Fig. 2. Secrecy outage probability with $1 / \beta$ under SC-SC scenario for $R_{s}=$ $0.1,2.0,1 / \beta_{s d}=6,9 \mathrm{~dB}, 1 / \alpha_{s e}=6,9 \mathrm{~dB}, 1 / \alpha=9 \mathrm{~dB}$ and $\gamma_{t h}=3$ $\mathrm{dB}$ of cognitive relay system.

used at $S D$ and MRC is used at $S E$. At lower secrecy rates, the performance of the MRC-MRC system and SC-SC system is comparable. However, at higher secrecy rates, SC-SC system performs better than the MRC-MRC system. This is because at higher secrecy rates, when both the destination and eavesdropper employs the same diversity scheme, the secrecy performance of the system is dominated by the diversity scheme used at the eavesdropper. Also, it is shown that $P_{o}$ increases in function of the required secrecy rate $R_{s}$, which is intuitive.

Fig. 4 shows the $P_{o}$ for cognitive system under SC-SC scenario as given in (11), with total SNR $1 / \beta$. The figure is plotted with different $1 / \alpha_{s e}=0,6,12 \mathrm{~dB}, 1 / \beta_{s d}=3,9 \mathrm{~dB}$ and fixed $R_{s}=1.0, \gamma_{t h}=3 \mathrm{~dB}, 1 / \alpha_{r e}=1 / \alpha=3 \mathrm{~dB}, I_{p}=9 \mathrm{~dB}$, $1 / \theta_{s p}=3 \mathrm{~dB}, 1 / \theta_{r p}=3 \mathrm{~dB}$. It can be observed from the plot that the secrecy performance is improved by increasing $1 / \beta_{s d}$ from $3 \mathrm{~dB}$ to $9 \mathrm{~dB}$ for a given $1 / \alpha_{\text {se }}=0$ or 6 or $12 \mathrm{~dB}$. It is however interesting to note that when the direct $S S-S E$ link quality is low, i.e., $1 / \alpha_{s e}=0 \mathrm{~dB}$, the amount of improvement in the secrecy performance by improving direct $S S-S D$ link quality is higher. Thus, when there is already low secrecy due to good quality channel of the eavesdropper, it intuitively becomes difficult to improve the secrecy of the system.

Fig. 5 shows the effect of $\gamma_{t h}$ on $P_{o}$, as given in (11), (13), (15) and (17). The figure has been plotted with different $1 / \alpha_{r e}=1 / \alpha=6,12 \mathrm{~dB}$ and fixed $R_{s}=1.0,1 / \beta_{s r}=$ $1 / \beta_{r d}=25 \mathrm{~dB}, 1 / \alpha_{s e}=3 \mathrm{~dB}, 1 / \beta_{s d}=3 \mathrm{~dB}, I_{p}=9 \mathrm{~dB}$ $, 1 / \theta_{s p}=3 \mathrm{~dB}, 1 / \theta_{r p}=3 \mathrm{~dB}$. It can be observed from the plot that when $\gamma_{t h}$ tends to zero, all curves saturate to a particular value depicted by using horizontal dashed line, which can be evaluated by substituting $\gamma_{t h}=0$ in (11), (13), (15) and (17). This shows that a fixed least $P_{o}$ could be achieved depending on the input parameters, when the message is perfectly decoded by the relay. When $\gamma_{t h}$ tends to infinity, due to high SNR threshold relay cannot decode the message, and as both $S S-S E$ and $S S-S D$ links exist, $P_{o}$ tends to $\left(1-\frac{\alpha_{s e} \theta_{s p}}{\left(\rho \beta_{s d}+\alpha_{s e}\right)\left(\theta_{s p}+\left(\frac{\rho-1}{I_{p}}\right) \beta_{s d}\right)}\right)$. This shows that the direct

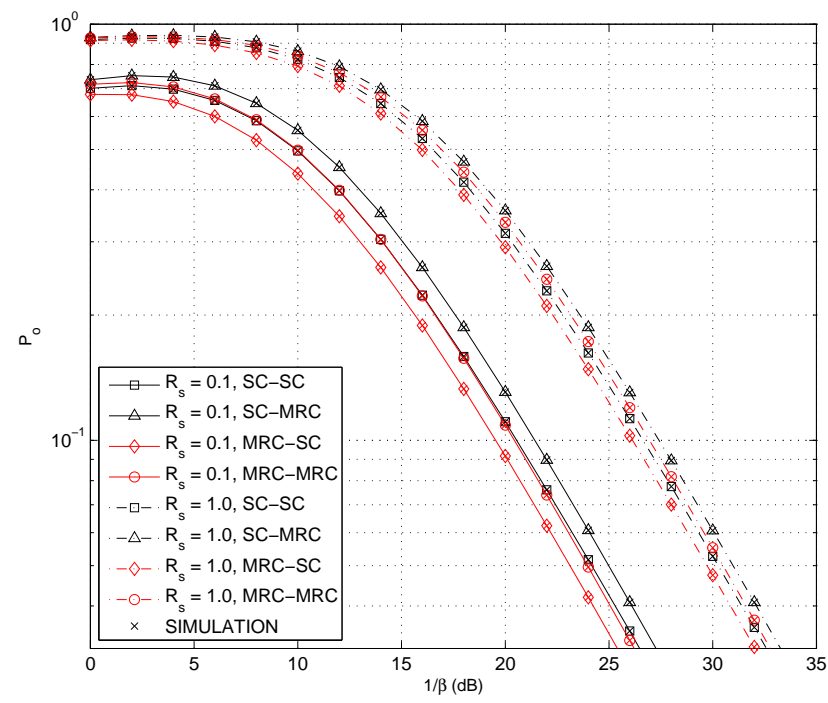

Fig. 3. Comparative analysis of $P_{o}$ with $1 / \beta$ under four scenarios for $R_{s}=$ $1.0,0.1,1 / \alpha=9 \mathrm{~dB}$ and $\gamma_{t h}=3 \mathrm{~dB}$ of cognitive system.

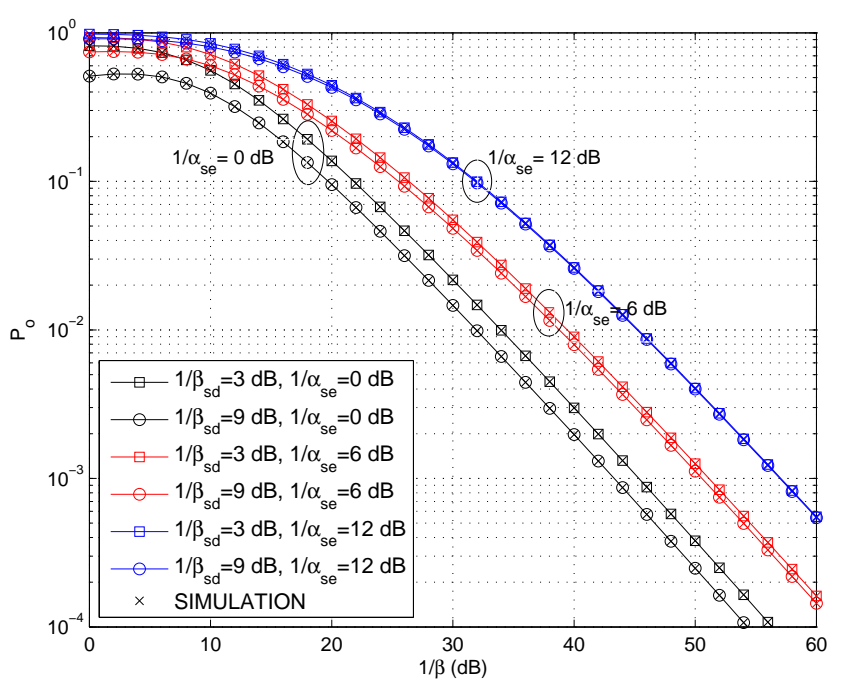

Fig. 4. Secrecy outage probability with $1 / \beta$ under SC-SC scenario for $1 / \beta_{s d}=$ $3,9 \mathrm{~dB}, 1 / \alpha_{s e}=0,6,12 \mathrm{~dB}, R_{s}=1.0,1 / \alpha=9 \mathrm{~dB}$ and $\gamma_{t h}=3 \mathrm{~dB}$ of cognitive relay system.

links have a significant impact on the system secrecy. Also, it is observed that $P_{o}$ increases in function of $1 / \alpha_{r e}$.

Fig. 6 shows the $P_{o}$ for cognitive balanced system under SCSC scenario as given in (11), with $\gamma_{t h}$. The figure is plotted with different $1 / \beta_{s d}=3,40 \mathrm{~dB}, 1 / \beta_{s r}=1 / \beta_{r d}=3,40$ $\mathrm{dB}, R_{s}=0.1,1.0$ and fixed $\gamma_{t h}=3 \mathrm{~dB}, 1 / \alpha_{s e}=3 \mathrm{~dB}$, $1 / \alpha_{r e}=1 / \alpha=3 \mathrm{~dB}, I_{m}=9 \mathrm{~dB}, 1 / \theta_{s p}=3 \mathrm{~dB}, 1 / \theta_{r p}=3$ $\mathrm{dB}$. It can be observed from the plot that as $\gamma_{t h}$ increases, the outage probability decreases when the quality of $S R-S D$ channel is very low in comparison to the quality of $S S-S D$ channel, i.e., $1 / \beta_{r d}=3 \mathrm{~dB}$ and $1 / \beta_{s d}=40 \mathrm{~dB}$. Whereas, the outage probability increases when the quality of $S R-S D$ channel is very high in comparison to the quality of $S S-S D$ channel, i.e., $1 / \beta_{r d}=40 \mathrm{~dB}$ and $1 / \beta_{s d}=3 \mathrm{~dB}$. This can be explained as follows. When $\gamma_{t h}$ increases, the probability of the relay to get 


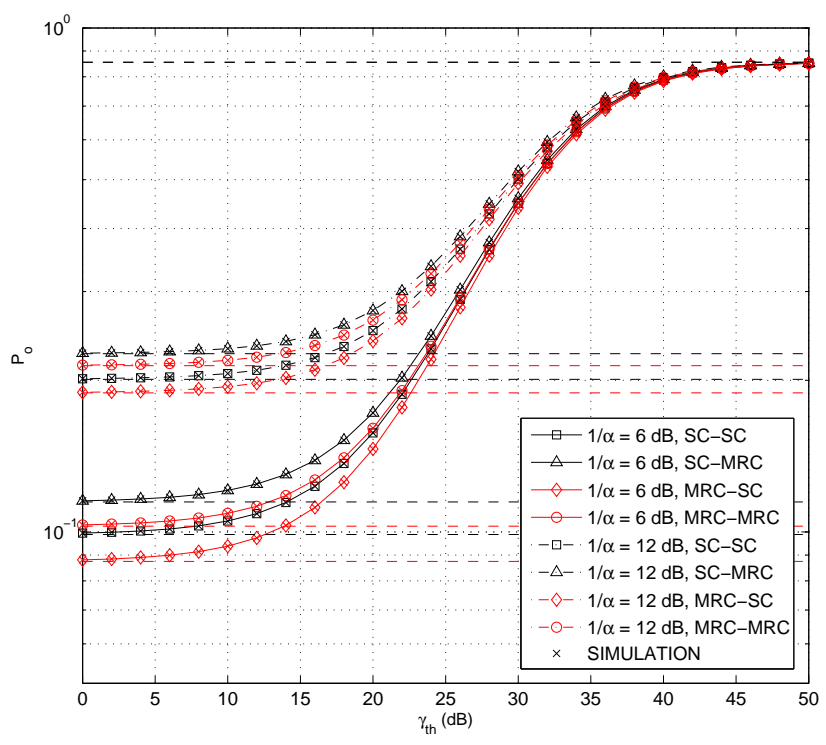

Fig. 5. Secrecy outage probability of the cognitive system with $\gamma_{t h}$ under four scenarios for $1 / \alpha=6,12 \mathrm{~dB}, R_{s}=1.0$.

selected decreases. Hence, when the quality of the $S S-S D$ channel is not good in comparison to $S R-S D$ channel, the SNR at the destination decreases and the secrecy outage probability increases. On the other hand, with an increase in $\gamma_{t h}$, there is decrease in the transmission towards the eavesdropper also, which decreases SNR at the eavesdropper. Thus, when the quality of $S S-S D$ channel is far better than the quality of $S R-S D$ channel, the SNR at the destination is increased. As a result, the secrecy outage probability decreases. It can also be observed from the plot that when $\gamma_{t h}$ tends to zero or infinity, all curves saturate to a particular value depicted by using horizontal dashed lines.

Fig. 7 shows $P_{o}$ for the cognitive system, as expressed in (11) for the SC-SC scenario, with $I_{p}$. The figure has been plotted with different $1 / \beta_{s r}=1 / \beta_{r d}=1 / \beta=25,30,35 \mathrm{~dB}$, $R_{s}=1.0,0.1$ and fixed $\gamma_{t h}=3 \mathrm{~dB}, 1 / \alpha_{r e}=1 / \alpha=9 \mathrm{~dB}$, $1 / \theta_{s p}=3 \mathrm{~dB}, 1 / \theta_{r p}=3 \mathrm{~dB}$. We can observe from the plot that $P_{o}$ decreases [3], with the increase in the $I_{p}$. This can be explained as with an increasing $I_{p}$, higher power can be transmitted by the SU, leading to a decrease of $P_{o}$. We can observe from the figure that when $I_{p}$ is increased beyond a certain value, $P_{o}$ for the SC-SC scenario converges to its respective $P_{o}$ floor [3]. For the other scenarios also, this observation holds true. It can be observed that the improvement in the main link average SNR, decreases the $P_{o}$ of cognitive relay system. $P_{o}$ increases in function of $R_{s}$, which is also intuitive.

\section{Conclusion}

The exact closed-form expressions are presented in this paper for the secrecy outage probability of threshold-based DF cognitive underlay relay system. We have assumed peak interference type of power control at the secondary relay and source. Unlike prior work, the relayed and the direct signals are combined at the eavesdropper and destination in our study and the secrecy performance with permutations of both MRC and SC diversity

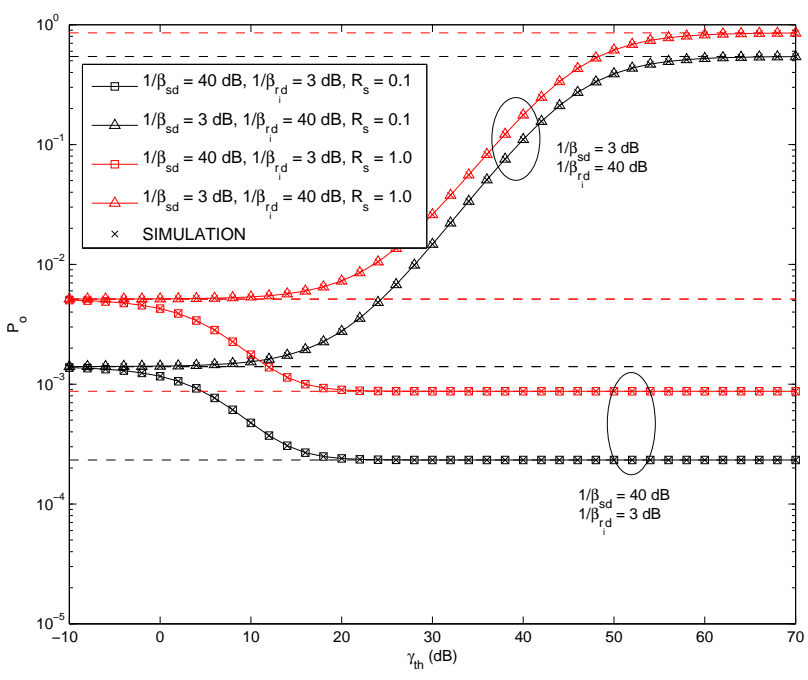

Fig. 6. Secrecy outage probability of the cognitive relay system with $\gamma_{t h}$ under SC-SC scenario for $1 / \beta_{s d}=3,40 \mathrm{~dB}, 1 / \beta_{s r}=1 / \beta_{r d}=3,40 \mathrm{~dB}$, $R_{s}=0.1,1.0,1 / \alpha=3 \mathrm{~dB}$.

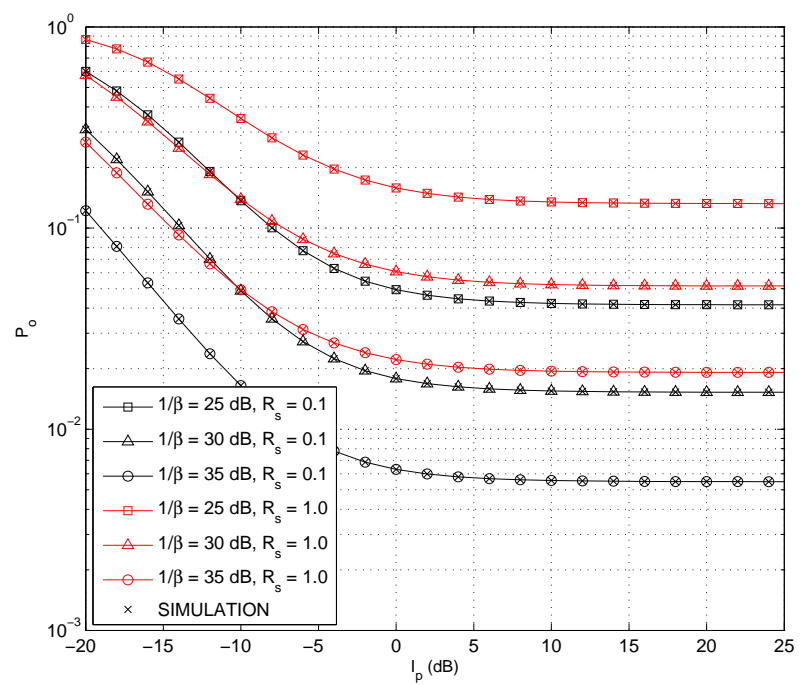

Fig. 7. Secrecy outage probability of the cognitive system with $I_{p}$ in SC-SC scenario for $1 / \beta=25,30,35 \mathrm{~dB}, R_{s}=0.1,1.0$ and $\gamma_{t h}=3 \mathrm{~dB}$.

schemes is investigated. Our results corroborate that MRC at $S D$ and SC at the eavesdropper gives the best performance. We also demonstrate that at at higher secrecy rates, SC-SC system performs better than the MRC-MRC system. We have shown that the secrecy performance of cognitive relay system is significantly affected by the threshold SNR, interference power, required secrecy rate and diversity scheme (MRC/SC). We also conclude that the direct link between source and eavesdropper plays a more crucial role in the secrecy performance of the system than between source and destination. Our research on analyzing the secrecy performance of this cognitive threshold-based relay system with the effect of channel knowledge at the transmitter is ongoing. Furthermore, in future this work can also be extended for multi-eavesdropper and multi-destination system. 


\section{Appendix}

A. Some standard integrals used for evaluation of expressions

The exponential random variables $\left|h_{s p}\right|^{2}$ and $\left|h_{r p}\right|^{2}$ are represented as $X$ and $Y$ for convenience. We have used the integration of the product of exponential integral function $\operatorname{Ei}(\cdot)$, where $\operatorname{Ei}(\mathrm{x})=\int_{-\infty}^{\mathrm{x}} \frac{\mathrm{e}^{\mathrm{t}}}{\mathrm{t}} \mathrm{dt}$ with exponential function and powers [36], given as

$$
\begin{aligned}
& \int_{0}^{\infty} x e^{-a x} E_{i}(b x) d x=\frac{-1}{a^{2}}\left[\ln \left(\frac{a}{b}-1\right)-\left(\frac{a}{a-b}\right)\right] \\
& \int_{0}^{\infty} x e^{-a x} E_{1}(b x) d x=\frac{1}{a^{2}}\left[\ln \left(1+\frac{a}{b}\right)-\left(\frac{a}{a+b}\right)\right]
\end{aligned}
$$

B. Evaluation of $Q_{1}$ in (11)

$$
\begin{aligned}
\mathbb{E}_{X, Y}\left[e^{-\frac{\gamma_{t h} \beta_{s r} X}{I_{p}}}\right] & =\mathbb{E}_{Y}\left[\int_{0}^{+\infty}\left(e^{-\frac{\gamma_{t h} \beta_{s r} x}{I_{p}}}\right) \theta_{s p} e^{-\theta_{s p} x}\right] \\
& =\left(\frac{\theta_{s p}}{\theta_{s p}+\frac{\gamma_{t h} \beta_{s r}}{I_{p}}}\right)
\end{aligned}
$$

C. Evaluation of $Q_{2}$ in (11)

$$
\begin{aligned}
& \mathbb{E}_{X, Y}\left[\frac{e^{-\frac{\gamma_{t h} \beta_{s r} X}{I_{p}}} \alpha_{r e} Y e^{-\left(\frac{\rho-1}{I_{p}}\right) \beta_{s d} X}}{\left(\rho \beta_{s d} X+\alpha_{r e} Y\right)}\right]= \\
& \int_{0}^{+\infty} \int_{0}^{+\infty}\left(\frac{e^{-\frac{\gamma_{t h} \beta_{s r} x}{I_{p}}} \alpha_{r e} y e^{-\left(\frac{\rho-1}{I_{p}}\right) \beta_{s d} x}}{\left(\rho \beta_{s d} x+\alpha_{r e} y\right)}\right) \\
& \quad \times \theta_{s p} e^{-\theta_{s p} x} \theta_{r p} e^{-\theta_{r p} y} d x d y \\
& =\int_{0}^{+\infty}\left(\frac{\alpha_{r e} \theta_{s p} \theta_{r p}}{\rho \beta_{s d}}\right) y e^{-y\left(\theta_{r p}-\frac{\alpha_{r e} z_{q}}{\beta_{s d} \rho}\right)} \\
& \quad \times E_{1}\left(\frac{\alpha_{r e} z_{q} y}{\beta_{s d} \rho}\right) d y
\end{aligned}
$$

where $z_{q}=\left(\frac{\rho-1}{I_{p}}\right) \beta_{s d}+\theta_{s p}+\frac{\gamma_{t h} \beta_{s r}}{I_{p}}$

D. Evaluation of $Q_{3}$ in (11)

$$
\begin{aligned}
& \mathbb{E}_{X, Y}\left[\frac{e^{-\frac{\gamma_{t h} \beta_{s r} X}{I_{p}}} \alpha_{s e} X e^{-\left(\frac{\rho-1}{I_{p}}\right) \beta_{s d} X}}{\left(\rho \beta_{s d} X+\alpha_{s e} X\right)}\right]= \\
& \int_{0}^{+\infty} \int_{0}^{+\infty}\left(\frac{e^{-\frac{\gamma_{t h} \beta_{s r} x}{I_{p}}} \alpha_{s e} x e^{-\left(\frac{\rho-1}{I_{p}}\right) \beta_{s d} x}}{\left(\rho \beta_{s d} x+\alpha_{s e} x\right)}\right) \\
& \quad \times \theta_{s p} e^{-\theta_{s p} x} \theta_{r p} e^{-\theta_{r p} y} d x d y \\
& =\frac{\theta_{r p} \theta_{s p} \alpha_{s e}}{z_{q} \theta_{r p}\left(\beta_{s d} \rho+\alpha_{s e}\right)}
\end{aligned}
$$

where $z_{q}=\left(\frac{\rho-1}{I_{p}}\right) \beta_{s d}+\theta_{s p}+\frac{\gamma_{t h} \beta_{s r}}{I_{p}}$.
E. Evaluation of $Q_{4}$ in (11)

$$
\begin{aligned}
& \mathbb{E}_{X, Y}\left[\frac{e^{-\frac{\gamma_{t h} \beta_{s r} X}{I_{p}}}\left(\alpha_{s e} X+\alpha_{r e} Y\right) e^{-\left(\frac{\rho-1}{I_{p}}\right) \beta_{s d} X}}{\left(\rho \beta_{s d} X+\alpha_{s e} X+\alpha_{r e} Y\right)}\right]= \\
& \int_{0}^{+\infty} \int_{0}^{+\infty}\left(\frac{e^{-\frac{\gamma_{t h} \beta_{s r} x}{I_{p}}}\left(\alpha_{s e} x+\alpha_{r e} y\right) e^{-\left(\frac{\rho-1}{I_{p}}\right) \beta_{s d} x}}{\left(\rho \beta_{s d} x+\alpha_{s e} x+\alpha_{r e} y\right)}\right) \\
& \quad \times \theta_{s p} e^{-\theta_{s p} x} \theta_{r p} e^{-\theta_{r p} y} d x d y \\
& =\int_{0}^{+\infty}\left(\frac{\alpha_{s e} \theta_{s p} \theta_{r p}}{\alpha_{r e}}\right) x e^{-x\left(z_{q}-\frac{\theta_{r p}\left(\beta_{s d} \rho+\alpha_{s e}\right)}{\alpha_{r e}}\right)} \times \\
& E_{1}\left(\frac{\theta_{r p}\left(\beta_{s d} \rho+\alpha_{s e}\right) x}{\alpha_{r e}}\right) d x+\int_{0}^{+\infty}\left(\frac{\alpha_{r e} \theta_{s p} \theta_{r p}}{\beta_{s d} \rho+\alpha_{s e}}\right) \times \\
& y e^{-y\left(\theta_{r p}-\frac{z_{q} \alpha_{r e}}{\left(\beta_{s d} \rho+\alpha_{s e}\right)}\right)} E_{1}\left(\frac{z_{q} \alpha_{r e} y}{\left(\beta_{s d} \rho+\alpha_{s e}\right)}\right) d y
\end{aligned}
$$

where $z_{q}=\left(\frac{\rho-1}{I_{p}}\right) \beta_{s d}+\theta_{s p}+\frac{\gamma_{t h} \beta_{s r}}{I_{p}}$.

F. Evaluation of $Q_{8}$ in (11)

$$
\begin{aligned}
& \mathbb{E}_{X, Y}\left[\frac{e^{-\frac{\gamma_{t h} \beta_{s r} X}{I_{p}}} \alpha_{r e} Y e^{-\left(\frac{\rho-1}{I_{p}}\right)\left(\beta_{s d} X+\beta_{r d} Y\right)}}{\left(\rho\left(\beta_{s d} X+\beta_{r d} Y\right)+\alpha_{r e} Y\right)}\right]= \\
& \int_{0}^{+\infty} \int_{0}^{+\infty}\left(\frac{e^{-\frac{\gamma_{t h} \beta_{s r} x}{I_{p}}} \alpha_{r e} y e^{-\left(\frac{\rho-1}{I_{p}}\right)\left(\beta_{s d} x+\beta_{r d} y\right)}}{\left(\rho\left(\beta_{s d} x+\beta_{r d} y\right)+\alpha_{r e}\right)}\right) \\
& \quad \times \theta_{s p} e^{-\theta_{s p} x} \theta_{r p} e^{-\theta_{r p} y} d x d y \\
& =\int_{0}^{+\infty}\left(\frac{\alpha_{r e} \theta_{s p} \theta_{r p}}{\rho \beta_{s d}}\right) y e^{-y\left(z_{q_{2}}-\frac{z_{q_{1}\left(\beta_{r d} \rho+\alpha_{r e}\right)}}{\beta_{s d} \rho}\right)} \\
& \quad \times E_{1}\left(\frac{z_{q_{1}}\left(\beta_{r d} \rho+\alpha_{r e}\right) y}{\beta_{s d} \rho}\right) d y
\end{aligned}
$$

where $z_{q_{1}}=\left(\frac{\rho-1}{I_{p}}\right) \beta_{s d}+\theta_{s p}+\frac{\gamma_{t h} \beta_{s r}}{I_{p}}$ and $z_{q_{2}}=\left(\frac{\rho-1}{I_{p}}\right) \beta_{r d}+$ $\theta_{r p}$.

\section{G. Evaluation of $Q_{10}$ in (11)}

$\mathbb{E}_{X, Y}\left[\frac{e^{-\frac{\gamma_{t h} \beta_{s r} X}{I_{p}}}\left(\alpha_{s e} X+\alpha_{r e} Y\right) e^{-\left(\frac{\rho-1}{I_{p}}\right)\left(\beta_{s d} X+\beta_{r d} Y\right)}}{\left(\rho\left(\beta_{s d} X+\beta_{r d} Y\right)+\alpha_{s e} X+\alpha_{r e} Y\right)}\right]=$

$\int_{0}^{+\infty} \int_{0}^{+\infty}\left(\frac{e^{-\frac{\gamma_{t h} \beta_{s r} x}{I_{p}}}\left(\alpha_{s e} x+\alpha_{r e} y\right) e^{-\left(\frac{\rho-1}{I_{p}}\right)\left(\beta_{s d} x+\beta_{r d} y\right)}}{\left(\rho\left(\beta_{s d} x+\beta_{r d} y\right)+\alpha_{s e} x+\alpha_{r e} y\right)}\right)$

$$
\times \theta_{s p} e^{-\theta_{s p} x} \theta_{r p} e^{-\theta_{r p} y} d x d y
$$$$
=\int_{0}^{+\infty}\left(\frac{\alpha_{s e} \theta_{s p} \theta_{r p}}{\left(\beta_{r d} \rho+\alpha_{r e}\right)}\right) x e^{-x\left(z_{q_{1}}-\frac{z_{q_{2}\left(\beta_{s d} \rho+\alpha_{s e}\right)}}{\beta_{r d} \rho+\alpha_{r e}}\right)} \times
$$$$
E_{1}\left(\frac{z_{q_{2}}\left(\beta_{s d} \rho+\alpha_{s e}\right) x}{\beta_{r d} \rho+\alpha_{r e}}\right) d x+\int_{0}^{+\infty}\left(\frac{\alpha_{r e} \theta_{s p} \theta_{r p}}{\beta_{s d} \rho+\alpha_{s e}}\right) \times
$$$$
y e^{-y\left(z_{q_{2}}-\frac{z_{q_{1}\left(\beta_{r d} \rho+\alpha_{r e}\right)}}{\beta_{s d^{\rho+\alpha_{s e}}}}\right)} E_{1}\left(\frac{z_{q_{1}}\left(\beta_{r d} \rho+\alpha_{r e}\right) y}{\beta_{s d} \rho+\alpha_{s e}}\right) d y
$$ 
where $z_{q_{1}}=\left(\frac{\rho-1}{I_{p}}\right) \beta_{s d}+\theta_{s p}+\frac{\gamma_{t h} \beta_{s r}}{I_{p}}$ and $z_{q_{2}}=\left(\frac{\rho-1}{I_{p}}\right) \beta_{r d}+$ $\theta_{r p}$.

\section{H. Evaluation of $Q_{11}$ in (11)}

$$
\begin{aligned}
& \mathbb{E}_{X, Y}\left[\left(1-\frac{\alpha_{s e} e^{-\left(\frac{\rho-1}{I_{p}}\right) \beta_{s d} X}}{\rho \beta_{s d}+\alpha_{s e}}\right)\left(1-e^{-\frac{\gamma_{t h} \beta_{s r} X}{I_{p}}}\right)\right]= \\
& \mathbb{E}_{Y}\left[\int _ { 0 } ^ { + \infty } \left(\left(1-\frac{\alpha_{s e} e^{-\left(\frac{\rho-1}{I_{p}}\right) \beta_{s d} x}}{\rho \beta_{s d}+\alpha_{s e}}\right) \times\right.\right. \\
& \left.\left.\quad\left(1-e^{-\frac{\gamma_{t h} \beta_{s r} x}{I_{p}}}\right)\right) \theta_{s p} e^{-\theta_{s p} x}\right] \\
& =\frac{\theta_{s p}}{\theta_{s p}+\frac{\gamma_{t h} \beta_{s r}}{I_{p}}} \\
& +\frac{\alpha_{s e} \theta_{s p}}{\left(\rho \beta_{s d}+\alpha_{s e}\right)\left(\theta_{s p}+\left(\frac{\rho-1}{I_{p}}\right) \beta_{s d}\right)} \\
& +\frac{\alpha_{s e} \theta_{s p}}{\left(\rho \beta_{s d}+\alpha_{s e}\right)\left(\theta_{s p}+\frac{\gamma_{t h} \beta_{s r}}{I_{p}}+\left(\frac{\rho-1}{I_{p}}\right) \beta_{s d}\right)}
\end{aligned}
$$

The other expressions for $Q_{5}, Q_{6}, Q_{7}$ and $Q_{9}$ can be readily obtained by interchanging $\beta_{s d} \leftrightarrow \beta_{r d}, \alpha_{s e} \leftrightarrow \alpha_{r e}$, and $\theta_{s p} \leftrightarrow \theta_{r p}$, in $Q_{3}, Q_{2}, Q_{4}$ and $Q_{8}$ respectively, while keeping the probability of selection term, i.e. $e^{-\frac{\gamma_{t h} \beta_{s r} \gamma_{s p}}{I_{p}}}$ intact. The expressions are omitted due to space limitations.

\section{Evaluation of $R_{4}$ in (13)}

$\mathbb{E}_{X, Y}\left[\frac{e^{-\frac{\gamma_{t h} \beta_{s r} X}{I_{p}}} \alpha_{r e} Y \alpha_{s e} X e^{-\left(\frac{\rho-1}{I_{p}}\right) \beta_{r d} Y}}{\left(\rho \beta_{r d} Y+\alpha_{s e} X\right)\left(\alpha_{r e} Y-\alpha_{s e} X\right)}\right]=$

$\int_{0}^{+\infty} \int_{0}^{+\infty}\left(\frac{e^{-\frac{\gamma_{t h} \beta_{s r} x}{I_{p}}} \alpha_{r e} y \alpha_{s e} x e^{-\left(\frac{\rho-1}{I_{p}}\right) \beta_{r d} y}}{\left(\rho \beta_{r d} y+\alpha_{s e} x\right)\left(\alpha_{r e} y-\alpha_{s e} x\right)}\right)$

$\times \theta_{s p} e^{-\theta_{s p} x} \theta_{r p} e^{-\theta_{r p} y} d x d y$

$=\int_{0}^{+\infty}\left(\frac{\alpha_{r e} \theta_{s p} \theta_{r p} \alpha_{r e}}{\alpha_{s e}\left(\beta_{r d} \rho+\alpha_{r e}\right)}\right) y e^{-y\left(z_{r}+\frac{\left(\theta_{s p}+\frac{\gamma_{t h} \beta_{s r}}{I_{p}}\right) \alpha_{r e}}{\alpha_{s e}}\right)} \times$

$E_{i}\left(\frac{\left(\theta_{s p}+\frac{\gamma_{t h} \beta_{s r}}{I_{p}}\right) \alpha_{r e} y}{\alpha_{s e}}\right) d y-\int_{0}^{+\infty}\left(\frac{\alpha_{r e} \theta_{s p} \theta_{r p} \beta_{r d} \rho}{\alpha_{s e}\left(\beta_{r d} \rho+\alpha_{r e}\right)}\right) \times$

$y e^{-y\left(z_{r}-\frac{\left(\theta_{s p}+\frac{\gamma_{t h} \beta_{s r}}{I_{p}}\right) \beta_{r d} \rho}{\alpha_{s e}}\right)} E_{1}\left(\frac{\left(\theta_{s p}+\frac{\gamma_{t h} \beta_{s r}}{I_{p}}\right) \beta_{r d} \rho y}{\alpha_{s e}}\right) d y$

where $z_{r}=\left(\frac{\rho-1}{I_{p}}\right) \beta_{r d}+\theta_{r p}$.
J. Evaluation of $R_{5}$ in (13)

$$
\begin{aligned}
& \mathbb{E}_{X, Y}\left[\frac{e^{-\frac{\gamma_{t h} \beta_{s r} X}{I_{p}}} \alpha_{r e} Y \alpha_{s e} X e^{-\left(\frac{\rho-1}{I_{p}}\right) \beta_{r d} Y}}{\left(\rho \beta_{r d} Y+\alpha_{r e} Y\right)\left(\alpha_{s e} X-\alpha_{r e} Y\right)}\right]= \\
& \int_{0}^{+\infty} \int_{0}^{+\infty}\left(\frac{e^{-\frac{\gamma_{t h} \beta_{s r} x}{I_{p}}} \alpha_{r e} y \alpha_{s e} x e^{-\left(\frac{\rho-1}{I_{p}}\right) \beta_{r d} y}}{\left(\rho \beta_{r d} y+\alpha_{r e} y\right)\left(\alpha_{s e} x-\alpha_{r e} y\right)}\right) \\
& \quad \times \theta_{s p} e^{-\theta_{s p} x} \theta_{r p} e^{-\theta_{r p} y} d x d y \\
& =\int_{0}^{+\infty}\left(\frac{\alpha_{s e} \theta_{s p} \theta_{r p}}{\rho \beta_{r d}+\alpha_{r e}}\right) x e^{-x\left(\theta_{s p}+\frac{\gamma_{t h} \beta_{s r}}{I_{p}}+\frac{\alpha_{s e} z_{r}}{\alpha_{r e}}\right)} \\
& \quad \times E_{i}\left(\frac{\alpha_{s e} z_{r} x}{\alpha_{r e}}\right) d x
\end{aligned}
$$

where $z_{r}=\left(\frac{\rho-1}{I_{p}}\right) \beta_{r d}+\theta_{r p}$.

K. Evaluation of $R_{7}$ in (13)

$\mathbb{E}_{X, Y}\left[\frac{e^{-\frac{\gamma_{t h} \beta_{s r} X}{I_{p}}} \alpha_{r e} Y \alpha_{s e} X e^{-\left(\frac{\rho-1}{I_{p}}\right)\left(\beta_{s d} X+\beta_{r d} Y\right)}}{\left(\rho\left(\beta_{s d} X+\beta_{r d} Y\right)+\alpha_{r e} Y\right)\left(\alpha_{s e} X-\alpha_{r e} Y\right)}\right]=$

$\int_{0}^{+\infty} \int_{0}^{+\infty}\left(\frac{e^{-\frac{\gamma_{t h} \beta_{s r} x}{I_{p}}} \alpha_{r e} y \alpha_{s e} x e^{-\left(\frac{\rho-1}{I_{p}}\right)\left(\beta_{s d} x+\beta_{r d} y\right)}}{\left(\rho\left(\beta_{s d} x+\beta_{r d} y\right)+\alpha_{r e} y\right)\left(\alpha_{s e} x-\alpha_{r e} y\right)}\right)$

$\times \theta_{s p} e^{-\theta_{s p} x} \theta_{r p} e^{-\theta_{r p} y} d x d y$

$=\int_{0}^{+\infty}\left(\frac{\alpha_{s e} \theta_{s p} \theta_{r p} \alpha_{s e}}{\beta_{r d} \rho \alpha_{s e}+\beta_{s d} \rho \alpha_{r e}+\alpha_{r e} \alpha_{s e}}\right) \times$

$x e^{-x\left(z_{r_{1}}+\frac{z_{r_{2}} \alpha_{s e}}{\alpha_{r e}}\right)} E_{i}\left(\frac{z_{r_{2}} \alpha_{s e} x}{\alpha_{r e}}\right) d x$

$-\int_{0}^{+\infty}\left(\frac{\alpha_{r e} \theta_{s p} \theta_{r p} \alpha_{s e} \beta_{s d} \rho}{\left(\beta_{r d} \rho \alpha_{s e}+\beta_{s d} \rho \alpha_{r e}+\alpha_{r e} \alpha_{s e}\right)\left(\beta_{r d} \rho+\alpha_{r e}\right)}\right)$

$x e^{-x\left(z_{r_{1}}-\frac{z_{r_{2}} \beta_{s d} \rho}{\beta_{r d} \rho+\alpha_{r e}}\right)} E_{1}\left(\frac{z_{r_{2}} \beta_{s d} \rho x}{\beta_{r d} \rho+\alpha_{r e}}\right) d x$

where $z_{r_{1}}=\left(\frac{\rho-1}{I_{p}}\right) \beta_{s d}+\theta_{s p}+\frac{\gamma_{t h} \beta_{s r}}{I_{p}}$ and $z_{r_{2}}=\left(\frac{\rho-1}{I_{p}}\right) \beta_{r d}+$ $\theta_{r p}$. The other expressions for $R_{2}, R_{3}$ and $R_{6}$ can be readily obtained by interchanging $\beta_{s d} \leftrightarrow \beta_{r d}, \alpha_{s e} \leftrightarrow \alpha_{r e}$, and $\theta_{s p} \leftrightarrow \theta_{r p}$, in $R_{5}, R_{4}$ and $R_{7}$ respectively, while keeping the probability of selection term, i.e. $e^{-\frac{\gamma_{t h} \beta_{s r} \gamma_{s p}}{I_{p}}}$ intact. $R_{1}$ and $R_{8}$ terms are similar to $Q_{1}$ and $Q_{11}$ respectively.

L. Evaluation of $S_{2}$ in (15)

$$
\begin{aligned}
& \mathbb{E}_{X, Y}\left[\frac{e^{-\frac{\gamma_{t h} \beta_{s r} X}{I_{p}}} \beta_{s d} X \alpha_{s e} X e^{-\left(\frac{\rho-1}{I_{p}}\right) \beta_{r d} Y}}{\left(\rho \beta_{r d} Y+\alpha_{s e} X\right)\left(\beta_{s d} X-\beta_{r d} Y\right)}\right]= \\
& \int_{0}^{+\infty} \int_{0}^{+\infty}\left(\frac{e^{-\frac{\gamma_{t h} \beta_{s r} x}{I_{p}}} \beta_{s d} x \alpha_{s e} x e^{-\left(\frac{\rho-1}{I_{p}}\right) \beta_{r d} y}}{\left(\rho \beta_{r d} y+\alpha_{s e} x\right)\left(\beta_{s d} x-\beta_{r d} y\right)}\right) \\
& \quad \times \theta_{s p} e^{-\theta_{s p} x} \theta_{r p} e^{-\theta_{r p} y} d x d y
\end{aligned}
$$




$$
\begin{aligned}
= & \int_{0}^{+\infty}\left(\frac{\beta_{s d} \alpha_{s e} \theta_{s p} \theta_{r p}}{\beta_{r d} \rho \beta_{s d}+\beta_{r d} \alpha_{s e}}\right) \times \\
& \left(x e^{-x\left(\theta_{s p}+\frac{\gamma_{t h} \beta_{s r}}{I_{p}}+\frac{z_{s} \beta_{s d}}{\beta_{r d}}\right)} E_{i}\left(\frac{z_{s} \beta_{s d} x}{\beta_{r d}}\right) d x\right. \\
& \left.+x e^{-x\left(\theta_{s p}+\frac{\gamma_{t h} \beta_{s r}}{I_{p}}-\frac{z_{s s} \alpha_{s e}}{\beta_{r d} \rho}\right)} E_{1}\left(\frac{z_{s} \alpha_{s e} x}{\beta_{r d} \rho}\right) d x\right)
\end{aligned}
$$

where $z_{s}=\left(\frac{\rho-1}{I_{p}}\right) \beta_{r d}+\theta_{r p}$.

\section{Evaluation of $S_{4}$ in (15)}

$$
\begin{aligned}
& \mathbb{E}_{X, Y}\left[\frac{e^{-\frac{\gamma_{t h} \beta_{s r} X}{I_{p}}} \beta_{s d} X \alpha_{r e} Y e^{-\left(\frac{\rho-1}{I_{p}}\right) \beta_{r d} Y}}{\left(\rho \beta_{r d} Y+\alpha_{r e} Y\right)\left(\beta_{s d} X-\beta_{r d} Y\right)}\right]= \\
& \int_{0}^{+\infty} \int_{0}^{+\infty}\left(\frac{e^{-\frac{\gamma_{t h} \beta_{s r} x}{I_{p}}} \beta_{s d} x \alpha_{r e} y e^{-\left(\frac{\rho-1}{I_{p}}\right) \beta_{r d} y}}{\left(\rho \beta_{r d} y+\alpha_{r e} y\right)\left(\beta_{s d} x-\beta_{r d} y\right)}\right) \\
& \quad \times \theta_{s p} e^{-\theta_{s p} x} \theta_{r p} e^{-\theta_{r p} y} d x d y \\
& =\int_{0}^{+\infty}\left(\frac{\alpha_{r e} \beta_{s d} \theta_{s p} \theta_{r p}}{\beta_{r d}\left(\rho \beta_{r d}+\alpha_{r e}\right)}\right) x e^{-x\left(\theta_{s p}+\frac{\gamma_{t h} \beta_{s r}}{I_{p}}+\frac{z_{s} \beta_{s d}}{\beta_{r d}}\right)} \\
& \quad \times E_{i}\left(\frac{z_{s} \beta_{s d} x}{\beta_{r d}}\right) d x
\end{aligned}
$$

where $z_{s}=\left(\frac{\rho-1}{I_{p}}\right) \beta_{r d}+\theta_{r p}$.

\section{N. Evaluation of $S_{6}$ in (15)}

$$
\begin{aligned}
& \mathbb{E}_{X, Y}\left[\frac{e^{-\frac{\gamma_{t h} \beta_{s r} X}{I_{p}}} \beta_{s d} X\left(\alpha_{r e} Y+\alpha_{s e} X\right) e^{-\left(\frac{\rho-1}{I_{p}}\right) \beta_{r d} Y}}{\left(\rho \beta_{r d} Y+\alpha_{s e} X+\alpha_{r e} Y\right)\left(\beta_{s d} X-\beta_{r d} Y\right)}\right]= \\
& \int_{0}^{+\infty} \int_{0}^{+\infty}\left(\frac{e^{-\frac{\gamma_{t h} \beta_{s r} x}{I_{p}}} \beta_{s d} x\left(\alpha_{r e} y+\alpha_{s e} x\right) e^{-\left(\frac{\rho-1}{I_{p}}\right) \beta_{r d} y}}{\left(\rho \beta_{r d} y+\alpha_{s e} x+\alpha_{r e} y\right)\left(\beta_{s d} x-\beta_{r d} y\right)}\right) \\
& \quad \times \theta_{s p} e^{-\theta_{s p} x} \theta_{r p} e^{-\theta_{r p} y} d x d y \\
& =\int_{0}^{+\infty}\left(\frac{\beta_{s d} \alpha_{s e} \theta_{s p} \theta_{r p}}{\beta_{r d} \rho \beta_{s d}+\beta_{r d} \alpha_{s e}+\beta_{s d} \alpha_{r e}}\right) \times \\
& \quad x e^{-x\left(\theta_{s p}+\frac{\gamma_{t h} \beta_{s r}}{I_{p}}+\frac{z_{s} \beta_{s d}}{\beta_{r d}}\right)_{E_{i}}\left(\frac{z_{s} \beta_{s d} x}{\beta_{r d}}\right) d x} \\
& \left.\quad+\int_{0}^{+\infty} x e^{-x\left(\theta_{s p}+\frac{\gamma_{t h} \beta_{s r}}{I_{p}}-\frac{z_{s s} \alpha_{s e}}{\beta_{r d} \rho+\alpha_{r e}}\right)} E_{1}\left(\frac{z_{s} \alpha_{s e} x}{\beta_{r d} \rho+\alpha_{r e}}\right) d x\right) \\
& \quad \int_{0}^{+\infty}\left(\frac{-\beta_{r d} \alpha_{r e} \theta_{s p} \theta_{r p}}{\beta_{r d} \rho \beta_{s d}+\beta_{r d} \alpha_{s e}+\beta_{s d} \alpha_{r e}}\right) \times \\
& \quad y e^{-y\left(z_{s}+\frac{\left(\theta_{s p}+\frac{\gamma_{t h} \beta_{s r}}{I_{p}}\right) \beta_{r d}}{\beta_{s d}}\right)} E_{E_{i}}\left(\frac{\left(\theta_{s p}+\frac{\gamma_{t h} \beta_{s r}}{I_{p}}\right) \beta_{r d} y}{\beta_{s d}}\right) d y \\
& \quad+\int_{0}^{+\infty}\left(\frac{\beta_{s d}\left(\alpha_{r e}+\beta_{r d} \rho\right) \alpha_{r e} \theta_{s p} \theta_{r p}}{\alpha_{s e}\left(\beta_{r d} \rho \beta_{s d}+\beta_{r d} \alpha_{s e}+\beta_{s d} \alpha_{r e}\right)}\right) \times
\end{aligned}
$$$$
\begin{aligned}
& y e^{-y\left(z_{s}-\frac{\left(\theta_{s p}+\frac{\gamma_{t h} \beta_{s r}}{I_{p}}\right)\left(\alpha_{r e}+\beta_{r d} \rho\right)}{\alpha_{s e}}\right)} \\
& \times E_{1}\left(\frac{\left(\theta_{s p}+\frac{\gamma_{t h} \beta_{s r}}{I_{p}}\right)\left(\alpha_{r e}+\beta_{r d} \rho\right) y}{\alpha_{s e}}\right) d y
\end{aligned}
$$

where $z_{s}=\left(\frac{\rho-1}{I_{p}}\right) \beta_{r d}+\theta_{r p}$. The other expressions for $S_{3}, S_{5}$ and $S_{7}$ can be readily obtained by interchanging $\beta_{s d} \leftrightarrow \beta_{r d}$, $\alpha_{s e} \leftrightarrow \alpha_{r e}$, and $\theta_{s p} \leftrightarrow \theta_{r p}$, in $S_{4}, S_{2}$ and $S_{6}$ respectively, while keeping the probability of selection term, i.e. $e^{-\frac{\gamma_{t h} \beta_{s r} \gamma_{s p}}{I_{p}}}$ intact. $S_{1}$ and $S_{8}$ terms are similar to $Q_{1}$ and $Q_{11}$ respectively.

\section{O. Evaluation of $T_{2}$ in (17)}

$$
\begin{aligned}
& \mathbb{E}_{X, Y}\left[\frac{e^{-\frac{\gamma_{t h} \beta_{s r} X}{I_{p}}} \beta_{s d} X \alpha_{r e} \alpha_{s e} X e^{-\left(\frac{\rho-1}{I_{p}}\right) \beta_{r d} Y}}{\left(\beta_{s d} X-\beta_{r d} Y\right)\left(\alpha_{s e} X-\alpha_{r e} Y\right)\left(\rho \beta_{r d}+\alpha_{r e}\right)}\right]= \\
& \int_{0}^{+\infty} \int_{0}^{+\infty}\left(\frac{e^{-\frac{\gamma_{t h} \beta_{s r} x}{I_{p}}} \beta_{s d} x \alpha_{r e} \alpha_{s e} x e^{-\left(\frac{\rho-1}{I_{p}}\right) \beta_{r d} y}}{\left(\beta_{s d} x-\beta_{r d} y\right)\left(\alpha_{s e} x-\alpha_{r e} y\right)\left(\rho \beta_{r d}+\alpha_{r e}\right)}\right) \\
& \quad \times \theta_{s p} e^{-\theta_{s p} x} \theta_{r p} e^{-\theta_{r p} y} d x d y \\
& =\int_{0}^{+\infty}\left(\frac{-\beta_{s d} \alpha_{s e} \alpha_{r e} \theta_{s p} \theta_{r p}}{\left(\rho \beta_{r d}+\alpha_{r e}\right)\left(\beta_{s d} \alpha_{r e}-\alpha_{s e} \beta_{r d}\right)}\right) \times \\
& \quad\left(x e^{-x\left(\left(\theta_{s p}+\frac{\gamma_{t h} \beta_{s r}}{I_{p}}\right)+\frac{z_{t_{1}} \beta_{s d}}{\beta_{r d}}\right)} E_{i}\left(\frac{z_{t_{1}} \beta_{s d} x}{\beta_{r d}}\right) d x\right. \\
& \left.-x e^{-x\left(\left(\theta_{s p}+\frac{\gamma_{t h} \beta_{s r}}{I_{p}}\right)+\frac{z_{t_{1}} \alpha_{s e}}{\alpha_{r e}}\right)} E_{i}\left(\frac{z_{t_{1}} \alpha_{s e} x}{\alpha_{r e}}\right) d x\right)
\end{aligned}
$$

where $z_{t_{1}}=\left(\frac{\rho-1}{I_{p}}\right) \beta_{r d}+\theta_{r p}$.

P. Evaluation of $T_{3}$ in (17)

$\mathbb{E}_{X, Y}\left[\frac{e^{-\frac{\gamma_{t h} \beta_{s r} X}{I_{p}}} X Y \beta_{r d} \alpha_{r e} \alpha_{s e} Y e^{-\left(\frac{\rho-1}{I_{p}}\right) \beta_{s d} X}}{\left(\beta_{r d} Y-\beta_{s d} X\right)\left(\alpha_{s e} X-\alpha_{r e} Y\right)\left(\rho \beta_{s d} X+\alpha_{r e} Y\right)}\right]=$

$\int_{0}^{+\infty} \int_{0}^{+\infty}\left(\frac{e^{-\frac{\gamma_{t h} \beta_{s r x} x}{I_{p}}} x y \beta_{r d} \alpha_{r e} \alpha_{s e} y e^{-\left(\frac{\rho-1}{I_{p}}\right) \beta_{s d} x}}{\left(\beta_{r d} y-\beta_{s d} x\right)\left(\alpha_{s e} x-\alpha_{r e} y\right)\left(\rho \beta_{s d} x+\alpha_{r e} y\right)}\right)$ $\times \theta_{s p} e^{-\theta_{s p} x} \theta_{r p} e^{-\theta_{r p} y} d x d y$

$=\int_{0}^{+\infty}\left(\frac{-\beta_{s d}^{2} \alpha_{s e} \alpha_{r e} \theta_{s p} \theta_{r p}}{\left(\beta_{r d} \alpha_{s e}-\alpha_{r e} \beta_{s d}\right)\left(\rho \beta_{r d} \beta_{s d}+\alpha_{r e} \beta_{s d}\right)}\right) \times$ $x e^{-x\left(z_{t_{2}}+\frac{\theta_{r p} \beta_{s d}}{\beta_{r d}}\right)} E_{i}\left(\frac{\theta_{r p} \beta_{s d} x}{\beta_{r d}}\right) d x+$

$\int_{0}^{+\infty}\left(\frac{-\alpha_{s e}^{3} \beta_{r d} \theta_{s p} \theta_{r p}}{\left(\alpha_{r e} \beta_{s d}-\beta_{r d} \alpha_{s e}\right)\left(\rho \alpha_{r e} \beta_{s d}+\alpha_{r e} \alpha_{s e}\right)}\right) \times$ $x e^{-x\left(z_{t_{2}}+\frac{\theta_{r p} \alpha_{s e}}{\alpha_{r e}}\right)} E_{i}\left(\frac{\theta_{r p} \alpha_{s e} x}{\alpha_{r e}}\right) d x+$

$\int_{0}^{+\infty}\left(\frac{-\rho^{2} \beta_{s d}^{2} \alpha_{s e} \beta_{r d} \theta_{s p} \theta_{r p}}{\left(\beta_{s d}+\rho \beta_{r d} \beta_{s d}\right)\left(\alpha_{r e} \alpha_{s e}+\rho \alpha_{r e} \beta_{s d}\right)}\right) \times$ 


$$
x e^{-x\left(z_{t_{2}}-\frac{\theta_{r p} \rho \beta_{s d}}{\alpha_{r e}}\right)} E_{1}\left(\frac{\theta_{r p} \rho \beta_{s d} x}{\alpha_{r e}}\right) d x
$$

where $z_{t_{2}}=\left(\frac{\rho-1}{I_{p}}\right) \beta_{s d}+\theta_{s p}+\frac{\gamma_{t h} \beta_{s r}}{I_{p}}$. The other expressions for $T_{4}$ and $T_{5}$ can be readily obtained by interchanging $\beta_{s d} \leftrightarrow$ $\beta_{r d}, \alpha_{s e} \leftrightarrow \alpha_{r e}$, and $\theta_{s p} \leftrightarrow \theta_{r p}$, in $T_{3}$ and $T_{2}$ respectively, while keeping the probability of selection term, i.e. $e^{-\frac{\gamma_{t h} \beta_{s r} \gamma_{s p}}{I_{p}}}$ intact. $T_{1}$ and $T_{6}$ terms are similar to $Q_{1}$ and $Q_{11}$ respectively.

\section{REFERENCES}

[1] C. Wang and H.-M. Wang, "On the secrecy throughput maximization for MISO cognitive radio network in slow fading channels," IEEE Trans. on Information Forensics and Security, vol. 9, no. 11, pp. 1814-1827, 2014.

[2] L. Sibomana, H.-J. Zepernick, and H. Tran, "Achievable secrecy capacity in an underlay cognitive radio network," in Proc. IEEE Conference on Commun. and Network Security (CNS), 2014, pp. 1-6.

[3] Y. Zou, X. Li, and Y.-C. Liang, "Secrecy outage and diversity analysis of cognitive radio systems," IEEE J. on Sel. Areas in Commun., vol. 32 no. 11, pp. 2222-2236, 2014.

[4] Y. Zou, X. Wang, and W. Shen, "Physical-layer security with multiuser scheduling in cognitive radio networks," IEEE Trans. on Commun., vol. 61, no. 12, pp. 5103-5113, 2013.

[5] Z. Shu, Y. Yang, Y. Qian, and R. Q. Hu, "Impact of interference on secrecy capacity in a cognitive radio network," in Proc. IEEE Global Telecommun. Conference (GLOBECOM), 2011, pp. 1-6.

[6] P. N. Son and H. Y. Kong, "The underlay cooperative cognitive network with secure transmission," in Proc. IEEE 27th Biennial Symposium on Commun. (QBSC), 2014, pp. 164-167.

[7] K. Chopra, R. Bose, and A. Joshi, "Secrecy performance of thresholdbased decode-and-forward cooperative cognitive radio network," IET Commun., vol. 11, no. 9, pp. 1396-1406, 2017.

[8] D.-J. Li, "Outage probability of cognitive radio networks with relay selection," IET Commun., vol. 5, no. 18, pp. 2730-2735, 2011.

[9] N. T. Do and B. An, "Secure transmission using decode-and-forward protocol for underlay cognitive radio networks," in Proc. IEEE Seventh International Conference on Ubiquitous and Future Networks, 2015, pp. 914 918.

[10] S. Ghose, C. Kundu, and R. Bose, "Secrecy performance of dual-hop decode-and-forward relay system with diversity combining at the eavesdropper," IET Commun., vol. 10, no. 8, pp. 904-914, 2016.

[11] H. Zhao, H. Liu, Y. Liu, C. Tang, and G. Pan, "Physical layer security of maximal ratio combining in underlay cognitive radio unit over Rayleigh fading channels," in Proc. IEEE Int. Conf. on Commun. Software and Networks (ICCSN), 2015, pp. 201-205.

[12] C. Kundu, S. Ghose, and R. Bose, "Secrecy outage of dual-hop regenerative multi-relay system with relay selection," IEEE Trans. on Wireless Commun., vol. 14, no. 8, pp. 4614-4625, 2015.

[13] A. D. Wyner, "The wire-tap channel," The Bell System Technical Journal, vol. 54, no. 8, pp. 1355-1387, 1975.

[14] J. Hu and N. C. Beaulieu, "Performance analysis of decode-and-forward relaying with selection combining," IEEE Commun. Lett., vol. 11, no. 6, pp. 489-491, 2007.

[15] A. Jindal, C. Kundu, and R. Bose, "Secrecy outage of dual-hop AF relay system with relay selection without eavesdropper's CSI," IEEE Commun. Lett., vol. 18, no. 10, pp. 1759-1762, 2014

[16] T. Zhang, Y. Huang, Y. Cai, and W. Yang, "Secure transmission in spectrum sharing relaying networks with multiple antennas," IEEE Communications Letters, vol. 20, no. 4, pp. 824-827, 2016.

[17] L. Fan, S. Zhang, T. Q. Duong, and G. K. Karagiannidis, "Secure switchand-stay combining (sssc) for cognitive relay networks," IEEE Trans. on Commun., vol. 64, no. 1, pp. 70-82, 2016

[18] M.-N. Nguyen, N.-P. Nguyen, D. B. Da Costa, H.-K. Nguyen, and R. T. De Sousa, "Secure cooperative half-duplex cognitive radio networks with $k$-th best relay selection," IEEE Access, vol. 5, pp. 6678-6687, 2017.

[19] T. T. Duy and P. N. Son, "Secrecy performances of multicast underlay cognitive protocols with partial relay selection and without eavesdropperâĂŹs information," KSII Trans. on Internet and Information Systems (TIIS), vol. 9, no. 11, pp. 4623-4643, 2015.

[20] I. Krikidis, J. S. Thompson, and S. McLaughlin, "Relay selection for secure cooperative networks with jamming," IEEE Trans. on Wireless Commun., vol. 8, no. 10, pp. 5003-5011, 2009.

[21] I. Krikidis, "Opportunistic relay selection for cooperative networks with secrecy constraints," IET Commun., vol. 4, no. 15, pp. 1787-1791, 2010
[22] H. Hui, A. L. Swindlehurst, G. Li, and J. Liang, "Secure relay and jammer selection for physical layer security," IEEE Signal Processing Lett., vol. 22 , no. 8, pp. 1147-1151, 2015 .

[23] E. R. Alotaibi and K. A. Hamdi, "Relay selection for multi-destination in cooperative networks with secrecy constraints," in Proc. IEEE 80th Vehicular Technology Conference (VTC Fall), 2014, pp. 1-5.

[24] V. N. Q. Bao, N. Linh-Trung, and M. Debbah, "Relay selection schemes for dual-hop networks under security constraints with multiple eavesdroppers," IEEE Trans. on Wireless Commun., vol. 12, no. 12, pp. 6076-6085, 2013.

[25] L. Wang, K. J. Kim, T. Q. Duong, M. Elkashlan, and H. V. Poor, "Security enhancement of cooperative single carrier systems," IEEE Trans. on Information Forensics and Security, vol. 10, no. 1, pp. 90-103, 2015.

[26] K. Chopra, R. Bose, and A. Joshi, "Secrecy outage performance of cooperative relay network with diversity combining," in Proc. IEEE International Conference on Signal and Image Processing (ICSIP), 2017.

[27] W. Yang, X. Xu, Y. Cai, and B. Zheng, "Secrecy outage analysis for cooperative DF underlay CRNs with outdated CSI," in Proc. IEEE Wireless Commun. and Networking Conference (WCNC), 2014, pp. 416-421.

[28] T. Q. Duong, T. T. Duy, M. Elkashlan, N. H. Tran, and O. A. Dobre, "Secured cooperative cognitive radio networks with relay selection," in Proc. IEEE Global Commun. Conference (GLOBECOM), 2014, pp. 3074-3079.

[29] Y. Liu, L. Wang, T. T. Duy, M. Elkashlan, and T. Q. Duong, "Relay selection for security enhancement in cognitive relay networks," IEEE Wireless Commun. Lett., vol. 4, no. 1, pp. 46-49, 2015.

[30] L. Sibomana, H.-J. Zepernick, and H. Tran, "On physical layer security for reactive DF cognitive relay networks," in Proc. IEEE Globecom Workshops (GC Wkshps), 2014, pp. 1290-1295.

[31] P. Chakraborty and S. Prakriya, "Secrecy outage performance of a cooperative cognitive relay network," IEEE Commun. Lett., vol. 21, no. 2, pp. 326-329, 2017

[32] J. Lee, H. Wang, J. G. Andrews, and D. Hong, "Outage probability of cognitive relay networks with interference constraints," IEEE Trans. on Wireless Commun., vol. 10, no. 2, pp. 390-395, 2011.

[33] M. Elkashlan, L. Wang, T. Q. Duong, G. K. Karagiannidis, and A. Nallanathan, "On the security of cognitive radio networks," IEEE Trans. on Vehicular Technology, vol. 64, no. 8, pp. 3790-3795, 2015.

[34] J. Proakis, Digital Commun., 4th ed. New York, NY, USA: McGraw-Hill, 2001.

[35] F. S. Al-Qahtani, C. Zhong, and H. M. Alnuweiri, "Opportunistic relay selection for secrecy enhancement in cooperative networks," IEEE Trans. on Commun., vol. 63, no. 5, pp. 1756-1770, 2015.

[36] A. D. Polyanin and A. V. Manzhirov, Handbook of integral equations. CRC press, 1998.

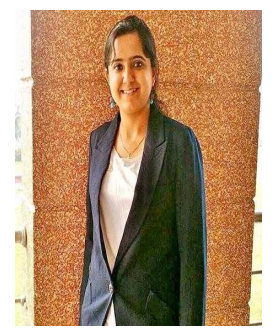

Khyati Chopra received her B.Tech degree in Electronics and Communication engineering from Indira Gandhi Institute of Technology (IGIT), GGSIPU, New Delhi, India in 2012. She received her M.Tech degree in Communication Engineering from Birla Institute of Technology and Science (BITS), Pilani, Rajasthan, India in 2014. Currently she is working towards her Ph.D. degree from Indian Institute of Technology (IIT), Delhi, India. Her research interests include cooperative communication, physical layer security, coding theory, cognitive radio networks and

game theory.

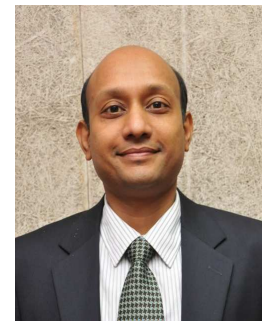

Ranjan Bose received the B.Tech. degree in electrical engineering from the Indian Institute of Technology (IIT) Kanpur, Kanpur, India, in 1992 and the M.S. and $\mathrm{Ph} . \mathrm{D}$. degrees in electrical engineering from the University of Pennsylvania, Philadelphia, PA, USA, in 1993 and 1995, respectively. From 1996 to 1997, he was with Alliance Semiconductor Inc., San Jose, CA, USA, as a Senior Design Engineer. Since November 1997, he has been with the Department of Electrical Engineering, IIT Delhi, New Delhi, India, where he is currently the Microsoft Chair Professor. His current research interests include broadband wireless access, wireless security, and coding theory. 


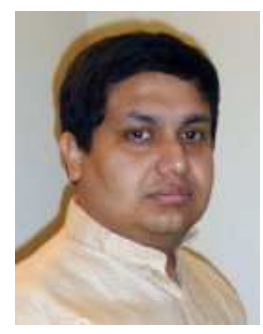

Anupam Joshi is a Professor of Computer Science and Electrical Engineering at UMBC. Earlier, he was an Assistant Professor in the CECS department at the University of Missouri, Columbia. He obtained a B. Tech degree in Electrical Engineering from IIT Delhi in 1989, and a Masters and Ph.D. in Computer Science from Purdue University in 1991 and 1993 respectively. His research interests are in the broad area of networked computing and intelligent systems. His primary focus has been on data management and security for mobile, pervasive, and sensor systems. He has created agent based middleware to support discovery, composition, and secure access of services/data over both infrastructure based and ad-hoc wireless networks, as well as systems that integrate sensors with the grid. He is also interested in Semantic Web, Social Media, and Data/Web Mining, where he has worked on creating personalized and secure web spaces using a combination of agents, policies, and soft computing. 